\title{
Suplementary material of the paper entitled:
}

\section{Theoretical Study of the Antioxidant Properties of Pyridoxine}

Jon M. Matxain, Mikael Ristila, Ake Strid and Leif A. Eriksson

Department of Natural Sciences and Orebro Life Sciences Center, Orebro University, 70182 Orebro, Sweden

In this supplementary material file we provide the Gibbs Free Energies in gas phase $\left(\mathrm{G}_{\mathrm{gas}}\right)$ and solution $\left(\mathrm{G}_{\mathrm{aq}}\right)$, and the coordinates for all characterized structures.

\section{Section 3.1. ADDITION REACTIONS}

\section{1. $\cdot$ OH addition}

\subsection{REACTANTS}

a) Pyridoxine

Energies: $\mathrm{G}_{\mathrm{gas}}=-591.770767 \quad \mathrm{G}_{\mathrm{aq}}=-591.967495$

Coordinates

\begin{tabular}{lrrr} 
& \multicolumn{1}{c}{$\mathrm{x}$} & \multicolumn{1}{c}{$\mathrm{y}$} & \multicolumn{1}{c}{$\mathrm{z}$} \\
$\mathrm{C}$ & 0.010624 & 2.001714 & 0.073482 \\
$\mathrm{C}$ & -1.859630 & 0.662745 & -0.067344 \\
$\mathrm{C}$ & -1.060173 & -0.492804 & 0.093001 \\
$\mathrm{C}$ & 0.325908 & -0.373582 & 0.268084 \\
$\mathrm{C}$ & 0.880258 & 0.923474 & 0.247645 \\
$\mathrm{C}$ & -3.349532 & 0.537076 & -0.231889 \\
$\mathrm{C}$ & 1.159865 & -1.613381 & 0.525282 \\
$\mathrm{C}$ & 2.365388 & 1.175548 & 0.387882 \\
$\mathrm{O}$ & 0.780951 & -2.721212 & -0.321003 \\
$\mathrm{O}$ & -1.697245 & -1.697936 & 0.088458 \\
$\mathrm{O}$ & 3.133284 & 0.631117 & -0.697116 \\
$\mathrm{~N}$ & -1.316995 & 1.883493 & -0.072135 \\
$\mathrm{H}$ & 0.402989 & 3.017553 & 0.053538 \\
$\mathrm{H}$ & -3.599145 & -0.088961 & -1.096098 \\
$\mathrm{H}$ & -3.804398 & 0.056403 & 0.642059 \\
$\mathrm{H}$ & -3.781910 & 1.530398 & -0.363276 \\
$\mathrm{H}$ & -1.030495 & -2.398743 & -0.054541 \\
$\mathrm{H}$ & 2.224816 & -1.405823 & 0.409043 \\
$\mathrm{H}$ & 0.986237 & -1.978769 & 1.544025
\end{tabular}


$\begin{array}{lrrr}\mathrm{H} & 1.091175 & -2.544588 & -1.219873 \\ \mathrm{H} & 2.543280 & 2.254822 & 0.482327 \\ \mathrm{H} & 2.775640 & 0.702345 & 1.285015 \\ \mathrm{H} & 2.838618 & 1.050425 & -1.516841\end{array}$

b) $\cdot \mathrm{OH}$ Radical

Energies: $\mathrm{G}_{\mathrm{gas}}=-75.746489 \quad \mathrm{G}_{\mathrm{aq}}=-75.784166$

$\underline{\text { Coordinates }}$

$\begin{array}{cccc} & \mathrm{x} & \mathrm{y} & \mathrm{z} \\ \mathrm{O} & 0.000000 & 0.000000 & 0.108834 \\ \mathrm{H} & 0.000000 & 0.000000 & -0.870670\end{array}$

\subsection{COMPLEXES}

a) $\mathrm{B}_{6}(\mathrm{~N} 1) \cdot \cdot \mathrm{OH}$

Energies: $\mathrm{G}_{\mathrm{gas}}=-667.513151 \quad \mathrm{G}_{\mathrm{aq}}=-667.743392$

Coordinates

$\begin{array}{lrrr} & & & \\ \mathrm{C} & 0.234492 & 1.181261 & 0.144020 \\ \mathrm{C} & 1.555479 & 0.673885 & 0.141814 \\ \mathrm{~N} & 1.761762 & -0.636380 & 0.271796 \\ \mathrm{C} & 0.735469 & -1.488516 & 0.367459 \\ \mathrm{C} & -0.595243 & -1.072022 & 0.379750 \\ \mathrm{C} & -0.857827 & 0.310524 & 0.275305 \\ \mathrm{C} & 2.735845 & 1.591658 & 0.000000 \\ \mathrm{O} & 3.541925 & -1.695814 & -0.699290 \\ \mathrm{C} & -1.698032 & -2.104669 & 0.479322 \\ \mathrm{O} & -2.526137 & -2.154082 & -0.691098 \\ \mathrm{C} & -2.263161 & 0.877040 & 0.347717 \\ \mathrm{O} & -2.474690 & 1.954317 & -0.589948 \\ \mathrm{O} & 0.089603 & 2.529529 & 0.029991 \\ \mathrm{H} & 0.994941 & -2.542435 & 0.426129 \\ \mathrm{H} & 2.597346 & 2.270721 & -0.847974 \\ \mathrm{H} & 2.850993 & 2.220558 & 0.890362 \\ \mathrm{H} & 3.638207 & 0.997765 & -0.150771 \\ \mathrm{H} & -0.837550 & 2.720233 & -0.218125 \\ \mathrm{H} & -3.011443 & 0.098469 & 0.192373 \\ \mathrm{H} & -2.433550 & 1.332445 & 1.330122 \\ \mathrm{H} & -2.552576 & 1.582178 & -1.479331 \\ \mathrm{H} & -1.256499 & -3.088590 & 0.683614 \\ \mathrm{H} & -2.385258 & -1.883493 & 1.301382\end{array}$


$\mathrm{H} \quad-1.980768 \quad-2.431181 \quad-1.439869$

$\mathrm{H} \quad 3.916087 \quad-1.708578 \quad 0.199955$

b) $\mathrm{B}_{6}(\mathrm{C} 2) \cdot \cdot \mathrm{OH}$ and $\mathrm{B}_{6}(\mathrm{C} 3) \cdot \cdot \mathrm{OH}$

Energies: $\mathrm{G}_{\mathrm{gas}}=-667.512727 \quad \mathrm{G}_{\mathrm{aq}}=-667.741905$

Coordinates

\begin{tabular}{lrrr} 
& \multicolumn{1}{c}{$\mathrm{x}$} & $\mathrm{y}$ & \multicolumn{1}{c}{$\mathrm{z}$} \\
$\mathrm{C}$ & -1.703674 & -0.640661 & 0.650192 \\
$\mathrm{~N}$ & -1.160529 & -1.855852 & 0.699541 \\
$\mathrm{C}$ & 0.138980 & -1.998414 & 0.384140 \\
$\mathrm{C}$ & 0.963722 & -0.961108 & -0.054330 \\
$\mathrm{C}$ & 0.410883 & 0.330680 & -0.143194 \\
$\mathrm{C}$ & -0.935732 & 0.497885 & 0.262744 \\
$\mathrm{C}$ & 2.400954 & -1.253375 & -0.427555 \\
$\mathrm{O}$ & 3.351945 & -0.577921 & 0.408475 \\
$\mathrm{C}$ & 1.174044 & 1.511466 & -0.705664 \\
$\mathrm{O}$ & 0.929407 & 2.732369 & 0.028033 \\
$\mathrm{O}$ & -1.524512 & 1.701510 & 0.379407 \\
$\mathrm{C}$ & -3.148744 & -0.474784 & 1.022364 \\
$\mathrm{O}$ & -1.269447 & 0.141248 & -2.147233 \\
$\mathrm{H}$ & 0.537054 & -3.008179 & 0.471453 \\
$\mathrm{H}$ & -3.243761 & 0.053057 & 1.979417 \\
$\mathrm{H}$ & -3.681319 & 0.127160 & 0.279093 \\
$\mathrm{H}$ & -3.613937 & -1.457774 & 1.113973 \\
$\mathrm{H}$ & -0.854019 & 2.399157 & 0.222964 \\
$\mathrm{H}$ & 2.245365 & 1.310685 & -0.743622 \\
$\mathrm{H}$ & 0.807885 & 1.709259 & -1.717922 \\
$\mathrm{H}$ & 1.439295 & 2.712565 & 0.849616 \\
$\mathrm{H}$ & 2.568872 & -2.338085 & -0.405511 \\
$\mathrm{H}$ & 2.625612 & -0.913176 & -1.442673 \\
$\mathrm{H}$ & 3.242550 & -0.896251 & 1.314644 \\
$\mathrm{H}$ & -1.651643 & -0.755240 & -2.099865
\end{tabular}

c) $\mathrm{B}_{6}(\mathrm{C} 4) \cdot \cdot \mathrm{OH}$ and $\mathrm{B}_{6}(\mathrm{C} 5) \cdot \cdot \mathrm{OH}$

Energies: $\mathrm{G}_{\mathrm{gas}}=-667.508383 \quad \mathrm{G}_{\mathrm{aq}}=-667.736925$

Coordinates

$\begin{array}{cccc} & & & \\ \mathrm{C} & -1.957399 & 0.580490 & -0.288801 \\ \mathrm{~N} & -1.475439 & 1.811051 & -0.151981 \\ \mathrm{C} & -0.157427 & 1.973696 & 0.095133 \\ \mathrm{C} & 0.745533 & 0.932442 & 0.238357\end{array}$




$\begin{array}{lrrr}\text { C } & 0.261993 & -0.406019 & 0.142655 \\ \mathrm{C} & -1.111409 & -0.557361 & -0.168488 \\ \mathrm{C} & 2.208561 & 1.222157 & 0.461598 \\ \mathrm{O} & 3.023162 & 0.845680 & -0.671656 \\ \mathrm{C} & 1.226944 & -1.579743 & 0.085608 \\ \mathrm{O} & 1.803289 & -1.733254 & -1.205163 \\ \mathrm{O} & -0.170508 & -0.968749 & 2.275344 \\ \mathrm{O} & -1.703729 & -1.752689 & -0.371672 \\ \mathrm{C} & -3.419712 & 0.404274 & -0.591186 \\ \mathrm{H} & 0.179195 & 3.005285 & 0.183181 \\ \mathrm{H} & -3.564460 & -0.100651 & -1.553219 \\ \mathrm{H} & -3.907038 & -0.219981 & 0.166276 \\ \mathrm{H} & -3.899286 & 1.383751 & -0.621715 \\ \mathrm{H} & -1.082047 & -2.475487 & -0.206082 \\ \mathrm{H} & 1.991228 & -1.457467 & 0.859201 \\ \mathrm{H} & 0.723089 & -2.521376 & 0.321030 \\ \mathrm{H} & 2.425626 & -0.993965 & -1.316626 \\ \mathrm{H} & 2.349833 & 2.285419 & 0.688261 \\ \mathrm{H} & 2.607283 & 0.646651 & 1.300515 \\ \mathrm{H} & 2.824010 & 1.447022 & -1.402691 \\ \mathrm{H} & -0.719568 & -0.224072 & 2.581646\end{array}$

d) $\mathrm{B}_{6}(\mathrm{C} 6)-\cdot \mathrm{OH}$

Energies: $\mathrm{G}_{\mathrm{gas}}=-667.511063 \quad \mathrm{G}_{\mathrm{aq}}=-667.740982$

$\underline{\text { Coordinates }}$

\begin{tabular}{lrrr} 
& \multicolumn{1}{c}{$\mathrm{x}$} & $\mathrm{y}$ & \multicolumn{1}{c}{$\mathrm{z}$} \\
$\mathrm{C}$ & 1.178368 & 0.561773 & 0.048251 \\
$\mathrm{C}$ & 1.853563 & -0.632073 & -0.329863 \\
$\mathrm{~N}$ & 1.179879 & -1.742111 & -0.626413 \\
$\mathrm{C}$ & -0.163810 & -1.720452 & -0.558277 \\
$\mathrm{C}$ & -0.914532 & -0.594272 & -0.175598 \\
$\mathrm{C}$ & -0.219490 & 0.586701 & 0.143672 \\
$\mathrm{C}$ & 3.354149 & -0.655432 & -0.405422 \\
$\mathrm{C}$ & -2.424974 & -0.682062 & -0.143651 \\
$\mathrm{O}$ & -3.048877 & 0.217436 & -1.074201 \\
$\mathrm{C}$ & -0.920907 & 1.827517 & 0.660483 \\
$\mathrm{O}$ & -0.331993 & 3.047001 & 0.154998 \\
$\mathrm{O}$ & 1.951310 & 1.641692 & 0.322583 \\
$\mathrm{O}$ & -0.922385 & -2.374076 & 1.716570 \\
$\mathrm{H}$ & -0.673167 & -2.631981 & -0.857426 \\
$\mathrm{H}$ & 3.723096 & 0.094664 & -1.114300 \\
$\mathrm{H}$ & 3.801464 & -0.410749 & 0.564981 \\
$\mathrm{H}$ & 3.683863 & -1.647196 & -0.718660 \\
$\mathrm{H}$ & 1.374767 & 2.434100 & 0.353985 \\
$\mathrm{H}$ & -1.985994 & 1.804709 & 0.426920
\end{tabular}




$\begin{array}{rrrr}\mathrm{H} & -0.804299 & 1.894182 & 1.748171 \\ \mathrm{H} & -0.612119 & 3.170877 & -0.762321 \\ \mathrm{H} & -2.734754 & -1.716456 & -0.326666 \\ \mathrm{H} & -2.810996 & -0.403424 & 0.839630 \\ \mathrm{H} & -2.843466 & -0.076190 & -1.971579 \\ \mathrm{H} & -0.016187 & -2.724387 & 1.804985\end{array}$

\subsection{TRANSITION STATES}

a) $\mathrm{B}_{6}(\mathrm{~N} 1) \cdot \cdot \mathrm{OH}$

Energies: $\mathrm{G}_{\mathrm{gas}}=-667.485767 \quad \mathrm{G}_{\mathrm{aq}}=-667.714203$

Coordinates

\begin{tabular}{lrrr} 
& \multicolumn{1}{c}{$\mathrm{x}$} & $\mathrm{y}$ & $\mathrm{z}$ \\
$\mathrm{C}$ & 1.693687 & 0.366877 & -0.233669 \\
$\mathrm{~N}$ & 1.670392 & -1.007519 & -0.298727 \\
$\mathrm{C}$ & 0.498625 & -1.697505 & -0.120246 \\
$\mathrm{C}$ & -0.694677 & -1.056734 & 0.153947 \\
$\mathrm{C}$ & -0.708434 & 0.355338 & 0.286513 \\
$\mathrm{C}$ & 0.509316 & 1.043209 & 0.061110 \\
$\mathrm{O}$ & 2.869441 & -1.700875 & 0.523221 \\
$\mathrm{C}$ & -1.954470 & -1.889073 & 0.268297 \\
$\mathrm{O}$ & -2.924830 & -1.576289 & -0.740145 \\
$\mathrm{C}$ & -1.927833 & 1.140358 & 0.699548 \\
$\mathrm{O}$ & -2.155407 & 2.289482 & -0.154789 \\
$\mathrm{O}$ & 0.563369 & 2.405958 & 0.106245 \\
$\mathrm{C}$ & 2.990113 & 1.063416 & -0.524357 \\
$\mathrm{H}$ & 0.580045 & -2.774479 & -0.220683 \\
$\mathrm{H}$ & 2.799267 & 2.042757 & -0.969833 \\
$\mathrm{H}$ & 3.575639 & 1.223279 & 0.388913 \\
$\mathrm{H}$ & 3.596431 & 0.455222 & -1.200034 \\
$\mathrm{H}$ & -0.343342 & 2.752285 & -0.017989 \\
$\mathrm{H}$ & -2.820892 & 0.513356 & 0.721627 \\
$\mathrm{H}$ & -1.790127 & 1.580317 & 1.694494 \\
$\mathrm{H}$ & -2.429044 & 1.969846 & -1.026282 \\
$\mathrm{H}$ & -1.694175 & -2.955036 & 0.238177 \\
$\mathrm{H}$ & -2.467015 & -1.707642 & 1.217555 \\
$\mathrm{H}$ & -2.541740 & -1.780166 & -1.604081 \\
$\mathrm{H}$ & 2.583664 & -1.568630 & 1.446104
\end{tabular}

b) $\mathrm{B}_{6}(\mathrm{C} 2)-\cdot \mathrm{OH}$

Energies: $\mathrm{G}_{\mathrm{gas}}=-667.509701 \quad \mathrm{G}_{\mathrm{aq}}=-667.739817$ 
$\underline{\text { Coordinates }}$

$\begin{array}{lrrr} & \mathrm{X} & \mathrm{y} & \mathrm{Z} \\ \mathrm{C} & 1.880564 & -0.381547 & -0.321444 \\ \mathrm{~N} & 1.354780 & -1.569075 & -0.582564 \\ \mathrm{C} & 0.011684 & -1.737720 & -0.442645 \\ \mathrm{C} & -0.877193 & -0.673317 & -0.126065 \\ \mathrm{C} & -0.334793 & 0.581289 & 0.155704 \\ \mathrm{C} & 1.062633 & 0.727689 & 0.042757 \\ \mathrm{O} & -0.164417 & -2.643786 & 1.402258 \\ \mathrm{C} & -2.355623 & -0.977972 & -0.019068 \\ \mathrm{O} & -3.162903 & -0.170761 & -0.891621 \\ \mathrm{C} & -1.176109 & 1.740778 & 0.653228 \\ \mathrm{O} & -0.743288 & 3.010825 & 0.114593 \\ \mathrm{O} & 1.691458 & 1.902109 & 0.276635 \\ \mathrm{C} & 3.369877 & -0.208073 & -0.433358 \\ \mathrm{H} & -0.388540 & -2.652013 & -0.867590 \\ \mathrm{H} & 3.622188 & 0.587701 & -1.142932 \\ \mathrm{H} & 3.804032 & 0.083257 & 0.530100 \\ \mathrm{H} & 3.816887 & -1.147542 & -0.762028 \\ \mathrm{H} & 1.017831 & 2.615333 & 0.302367 \\ \mathrm{H} & -2.232427 & 1.582074 & 0.433325 \\ \mathrm{H} & -1.056460 & 1.846228 & 1.737492 \\ \mathrm{H} & -1.048192 & 3.082849 & -0.800314 \\ \mathrm{H} & -2.521543 & -2.044576 & -0.205022 \\ \mathrm{H} & -2.721174 & -0.771234 & 0.990877 \\ \mathrm{H} & -2.975228 & -0.423081 & -1.805320 \\ \mathrm{H} & 0.746123 & -2.989326 & 1.397405\end{array}$

c) $\mathrm{B}_{6}(\mathrm{C} 3)-\cdot \mathrm{OH}$

Energies: $\mathrm{G}_{\mathrm{gas}}=-667.509297 \quad \mathrm{G}_{\mathrm{aq}}=-667.737590$

$\underline{\text { Coordinates }}$

$\begin{array}{crcc} & \mathrm{x} & \mathrm{y} & \mathrm{z} \\ \mathrm{C} & 1.862869 & -0.717396 & -0.241590 \\ \mathrm{~N} & 1.200939 & -1.872642 & -0.407732 \\ \mathrm{C} & -0.126944 & -1.868781 & -0.302265 \\ \mathrm{C} & -0.907500 & -0.724887 & 0.032747 \\ \mathrm{C} & -0.209876 & 0.512772 & 0.172474 \\ \mathrm{C} & 1.176263 & 0.496733 & 0.047228 \\ \mathrm{C} & -2.405069 & -0.760153 & -0.222501 \\ \mathrm{O} & -2.747985 & -0.086662 & -1.440660 \\ \mathrm{O} & -1.246777 & -1.310675 & 1.987893 \\ \mathrm{C} & -0.929361 & 1.790674 & 0.545169 \\ \mathrm{O} & -0.367150 & 2.952040 & -0.103126 \\ \mathrm{O} & 1.946297 & 1.608741 & 0.189261\end{array}$




$\begin{array}{lrrr}\mathrm{C} & 3.356967 & -0.725309 & -0.371397 \\ \mathrm{H} & -0.630031 & -2.822458 & -0.449311 \\ \mathrm{H} & 3.686632 & -0.019963 & -1.143019 \\ \mathrm{H} & 3.833526 & -0.402255 & 0.562013 \\ \mathrm{H} & 3.694448 & -1.731837 & -0.622683 \\ \mathrm{H} & 1.361789 & 2.393838 & 0.148742 \\ \mathrm{H} & -1.996105 & 1.725719 & 0.327328 \\ \mathrm{H} & -0.813620 & 1.979689 & 1.618148 \\ \mathrm{H} & -0.642728 & 2.950737 & -1.030376 \\ \mathrm{H} & -2.745911 & -1.801003 & -0.230183 \\ \mathrm{H} & -2.949028 & -0.246218 & 0.569173 \\ \mathrm{H} & -2.405460 & -0.593795 & -2.188469 \\ \mathrm{H} & -0.379262 & -1.653430 & 2.266621\end{array}$

d) $\mathrm{B}_{6}(\mathrm{C} 4) \cdot-\mathrm{OH}$

Energies: $\mathrm{G}_{\mathrm{gas}}=-667.506815 \quad \mathrm{G}_{\mathrm{aq}}=-667.527979$

Coordinates

\begin{tabular}{lrrr} 
& \multicolumn{1}{c}{$\mathrm{x}$} & $\mathrm{y}$ & $\mathrm{z}$ \\
$\mathrm{C}$ & -1.122095 & -0.560316 & -0.143221 \\
$\mathrm{C}$ & -1.962301 & 0.578925 & -0.279293 \\
$\mathrm{~N}$ & -1.474756 & 1.808970 & -0.158185 \\
$\mathrm{C}$ & -0.154813 & 1.969043 & 0.087235 \\
$\mathrm{C}$ & 0.744351 & 0.928012 & 0.241688 \\
$\mathrm{C}$ & 0.254810 & -0.413855 & 0.171070 \\
$\mathrm{C}$ & -3.425494 & 0.405575 & -0.579817 \\
$\mathrm{C}$ & 2.209173 & 1.213891 & 0.454553 \\
$\mathrm{O}$ & 3.013642 & 0.836881 & -0.686322 \\
$\mathrm{C}$ & 1.221572 & -1.585334 & 0.071657 \\
$\mathrm{O}$ & 1.758079 & -1.721226 & -1.236985 \\
$\mathrm{O}$ & -1.718818 & -1.756563 & -0.320995 \\
$\mathrm{O}$ & -0.082688 & -0.939711 & 2.244946 \\
$\mathrm{H}$ & 0.185383 & 3.000497 & 0.162034 \\
$\mathrm{H}$ & -3.572793 & -0.115690 & -1.532647 \\
$\mathrm{H}$ & -3.917019 & -0.201596 & 0.188753 \\
$\mathrm{H}$ & -3.899174 & 1.387193 & -0.628200 \\
$\mathrm{H}$ & -1.100064 & -2.477725 & -0.137407 \\
$\mathrm{H}$ & 2.007451 & -1.467811 & 0.823502 \\
$\mathrm{H}$ & 0.726429 & -2.530410 & 0.311816 \\
$\mathrm{H}$ & 2.385678 & -0.987074 & -1.352490 \\
$\mathrm{H}$ & 2.355060 & 2.276533 & 0.680991 \\
$\mathrm{H}$ & 2.611696 & 0.635865 & 1.289773 \\
$\mathrm{H}$ & 2.816933 & 1.446033 & -1.411509 \\
$\mathrm{H}$ & -0.629232 & -0.199303 & 2.564297
\end{tabular}


e) $\mathrm{B}_{6}(\mathrm{C} 5) \cdot \cdot \mathrm{OH}$

Energies: $\mathrm{G}_{\mathrm{gas}}=-667.506260 \quad \mathrm{G}_{\mathrm{aq}}=-667.736743$

Coordinates

\begin{tabular}{lrrr} 
& \multicolumn{1}{c}{$\mathrm{x}$} & $\mathrm{y}$ & $\mathrm{z}$ \\
$\mathrm{C}$ & -1.711488 & -0.783423 & 0.333308 \\
$\mathrm{~N}$ & -1.127999 & -1.973640 & 0.267219 \\
$\mathrm{C}$ & 0.188374 & -2.030925 & -0.000000 \\
$\mathrm{C}$ & 1.024870 & -0.914061 & -0.171957 \\
$\mathrm{C}$ & 0.455672 & 0.355296 & -0.085634 \\
$\mathrm{C}$ & -0.965846 & 0.430616 & 0.096972 \\
$\mathrm{C}$ & 2.502068 & -1.130071 & -0.429905 \\
$\mathrm{O}$ & 3.341522 & -0.600207 & 0.606481 \\
$\mathrm{C}$ & 1.223806 & 1.626213 & -0.356110 \\
$\mathrm{O}$ & 0.865320 & 2.694994 & 0.551432 \\
$\mathrm{O}$ & -1.578628 & 1.585369 & 0.456056 \\
$\mathrm{O}$ & -1.320228 & 0.389462 & -1.916998 \\
$\mathrm{C}$ & -3.170064 & -0.709882 & 0.672914 \\
$\mathrm{H}$ & 0.615507 & -3.031607 & -0.038221 \\
$\mathrm{H}$ & -3.325320 & -0.141309 & 1.597310 \\
$\mathrm{H}$ & -3.736177 & -0.194659 & -0.111018 \\
$\mathrm{H}$ & -3.560612 & -1.721237 & 0.793125 \\
$\mathrm{H}$ & -0.903233 & 2.294603 & 0.511971 \\
$\mathrm{H}$ & 2.300860 & 1.457304 & -0.323641 \\
$\mathrm{H}$ & 0.944859 & 1.994122 & -1.349501 \\
$\mathrm{H}$ & 1.283808 & 2.530268 & 1.407726 \\
$\mathrm{H}$ & 2.693760 & -2.201519 & -0.572351 \\
$\mathrm{H}$ & 2.824780 & -0.621799 & -1.342925 \\
$\mathrm{H}$ & 3.152044 & -1.074580 & 1.427148 \\
$\mathrm{H}$ & -2.142529 & 0.906372 & -1.858697
\end{tabular}

f) $\mathrm{B}_{6}(\mathrm{C} 6) \cdot \cdot \mathrm{OH}$

Energies: $\mathrm{G}_{\mathrm{gas}}=-667.505416 \quad \mathrm{G}_{\mathrm{aq}}=-667.737144$

$\underline{\text { Coordinates }}$

$\begin{array}{crrr} & & & \\ \mathrm{C} & -1.713570 & 0.431964 & -0.362289 \\ \mathrm{~N} & -1.256762 & 1.711414 & -0.440069 \\ \mathrm{C} & 0.024641 & 1.947887 & -0.191227 \\ \mathrm{C} & 0.978733 & 0.958985 & 0.124383 \\ \mathrm{C} & 0.556880 & -0.378391 & 0.164593 \\ \mathrm{C} & -0.792074 & -0.644835 & -0.130078 \\ \mathrm{C} & 2.411219 & 1.370414 & 0.386062 \\ \mathrm{O} & 3.335910 & 0.828141 & -0.568584\end{array}$




$\begin{array}{lrrr}\mathrm{C} & 1.472636 & -1.513307 & 0.579117 \\ \mathrm{O} & 1.271821 & -2.708254 & -0.208256 \\ \mathrm{O} & -1.287214 & -1.892473 & -0.191710 \\ \mathrm{C} & -3.081543 & 0.150102 & -0.918275 \\ \mathrm{O} & -2.243762 & 0.392669 & 1.673029 \\ \mathrm{H} & 0.337571 & 2.990474 & -0.245460 \\ \mathrm{H} & -3.004909 & -0.078906 & -1.989721 \\ \mathrm{H} & -3.543558 & -0.698968 & -0.415100 \\ \mathrm{H} & -3.706192 & 1.036711 & -0.799196 \\ \mathrm{H} & -0.542326 & -2.529963 & -0.170094 \\ \mathrm{H} & 2.520687 & -1.213154 & 0.538140 \\ \mathrm{H} & 1.235580 & -1.819307 & 1.604536 \\ \mathrm{H} & 1.666900 & -2.577500 & -1.081395 \\ \mathrm{H} & 2.478197 & 2.465859 & 0.409404 \\ \mathrm{H} & 2.762317 & 1.006112 & 1.356227 \\ \mathrm{H} & 3.115896 & 1.179252 & -1.442010 \\ \mathrm{H} & -2.278406 & 1.361913 & 1.765596\end{array}$

\subsection{PRODUCTS}

a) $\mathrm{B}_{6}(\mathrm{~N} 1) \cdot \cdot \mathrm{OH}$

Energies: $\mathrm{G}_{\mathrm{gas}}=-667.485659 \quad \mathrm{G}_{\mathrm{aq}}=-667.713757$

$\underline{\text { Coordinates }}$

$\begin{array}{lrrr} & \mathrm{x} & \mathrm{y} & \mathrm{z} \\ \mathrm{N} & -1.670321 & -1.024184 & 0.223835 \\ \mathrm{C} & -0.482134 & -1.713857 & 0.033809 \\ \mathrm{C} & 0.702813 & -1.053708 & -0.188334 \\ \mathrm{C} & 0.715305 & 0.367759 & -0.285665 \\ \mathrm{C} & -0.522169 & 1.045750 & -0.083957 \\ \mathrm{C} & -1.711437 & 0.375313 & 0.153538 \\ \mathrm{C} & -3.015688 & 1.046337 & 0.461098 \\ \mathrm{O} & -0.564992 & 2.412022 & -0.106387 \\ \mathrm{C} & 1.930635 & 1.167056 & -0.665579 \\ \mathrm{O} & 2.152261 & 2.291357 & 0.229846 \\ \mathrm{C} & 1.969377 & -1.875426 & -0.295891 \\ \mathrm{O} & 2.912469 & -1.589802 & 0.746017 \\ \mathrm{H} & -0.563827 & -2.791131 & 0.119729 \\ \mathrm{H} & -2.842819 & 2.103937 & 0.666940 \\ \mathrm{H} & -3.726764 & 0.973594 & -0.370011 \\ \mathrm{H} & -3.489441 & 0.576088 & 1.330487 \\ \mathrm{H} & 0.339872 & 2.746268 & 0.059158 \\ \mathrm{H} & 2.826451 & 0.544132 & -0.702662 \\ \mathrm{H} & 1.804210 & 1.646644 & -1.644235 \\ \mathrm{H} & 2.385527 & 1.938116 & 1.100422 \\ \mathrm{H} & 1.715775 & -2.943666 & -0.305615\end{array}$


$\begin{array}{lrrr}\mathrm{H} & 2.505087 & -1.659817 & -1.225054 \\ \mathrm{H} & 2.496308 & -1.793403 & 1.594640 \\ \mathrm{O} & -2.819535 & -1.699724 & -0.327737 \\ \mathrm{H} & -2.719960 & -1.637652 & -1.298684\end{array}$

b) $\mathrm{B}_{6}(\mathrm{C} 2)-\cdot \mathrm{OH}$

Energies: $\mathrm{G}_{\mathrm{gas}}=-667.534103 \quad \mathrm{G}_{\mathrm{aq}}=-667.761805$

$\underline{\text { Coordinates }}$

\begin{tabular}{lrrr} 
& \multicolumn{1}{c}{$\mathrm{x}$} & $\mathrm{y}$ & $\mathrm{z}$ \\
$\mathrm{N}$ & -1.466828 & -1.560627 & 0.363331 \\
$\mathrm{C}$ & -0.052257 & -1.832641 & 0.228539 \\
$\mathrm{C}$ & 0.876539 & -0.674534 & -0.040535 \\
$\mathrm{C}$ & 0.353965 & 0.586446 & -0.217549 \\
$\mathrm{C}$ & -1.049333 & 0.770114 & -0.066502 \\
$\mathrm{C}$ & -1.915787 & -0.347933 & 0.212419 \\
$\mathrm{C}$ & -3.398415 & -0.103724 & 0.328630 \\
$\mathrm{O}$ & -1.637575 & 1.986994 & -0.185505 \\
$\mathrm{C}$ & 1.204497 & 1.771235 & -0.636892 \\
$\mathrm{O}$ & 0.847478 & 2.986621 & 0.061565 \\
$\mathrm{C}$ & 2.353009 & -0.995321 & -0.115890 \\
$\mathrm{O}$ & 3.157501 & -0.222263 & 0.797586 \\
$\mathrm{H}$ & 0.261378 & -2.334735 & 1.164158 \\
$\mathrm{H}$ & -3.614340 & 0.614704 & 1.126913 \\
$\mathrm{H}$ & -3.796640 & 0.326230 & -0.597003 \\
$\mathrm{H}$ & -3.901529 & -1.048547 & 0.539471 \\
$\mathrm{H}$ & -0.939609 & 2.674222 & -0.135115 \\
$\mathrm{H}$ & 2.265955 & 1.562583 & -0.502489 \\
$\mathrm{H}$ & 1.020830 & 2.000154 & -1.692527 \\
$\mathrm{H}$ & 1.187983 & 2.936877 & 0.965321 \\
$\mathrm{H}$ & 2.502236 & -2.067493 & 0.053367 \\
$\mathrm{H}$ & 2.762372 & -0.772710 & -1.106381 \\
$\mathrm{H}$ & 2.918188 & -0.472369 & 1.699810 \\
$\mathrm{O}$ & 0.131068 & -2.770596 & -0.849673 \\
$\mathrm{H}$ & -0.620107 & -3.382438 & -0.803949
\end{tabular}

c) $\mathrm{B}_{6}(\mathrm{C} 3) \cdot \cdot \mathrm{OH}$

Energies: $\mathrm{G}_{\mathrm{gas}}=-667.528022 \quad \mathrm{G}_{\mathrm{aq}}=-667.755469$

$\underline{\text { Coordinates }}$

$\begin{array}{cccc} & \mathrm{x} & \mathrm{y} & \mathrm{z} \\ \mathrm{N} & -1.216849 & -1.878325 & 0.000000 \\ \mathrm{C} & 0.076372 & -1.937964 & -0.195640\end{array}$ 


$\begin{array}{lrrr}\mathrm{C} & 1.010087 & -0.771058 & -0.414091 \\ \mathrm{C} & 0.267395 & 0.533315 & -0.383181 \\ \mathrm{C} & -1.080871 & 0.543531 & -0.135017 \\ \mathrm{C} & -1.831244 & -0.669258 & 0.041051 \\ \mathrm{C} & -3.310168 & -0.628610 & 0.265764 \\ \mathrm{O} & -1.805003 & 1.698359 & -0.055583 \\ \mathrm{C} & 1.002905 & 1.822596 & -0.643604 \\ \mathrm{O} & 0.664889 & 2.849055 & 0.319154 \\ \mathrm{C} & 2.164203 & -0.821327 & 0.629973 \\ \mathrm{O} & 1.756054 & -0.501218 & 1.951516 \\ \mathrm{H} & 0.527406 & -2.933548 & -0.218026 \\ \mathrm{H} & -3.559144 & -0.037930 & 1.156156 \\ \mathrm{H} & -3.825819 & -0.144626 & -0.573303 \\ \mathrm{H} & -3.688738 & -1.645331 & 0.383803 \\ \mathrm{H} & -1.182606 & 2.440334 & 0.078856 \\ \mathrm{H} & 2.085277 & 1.669310 & -0.675183 \\ \mathrm{H} & 0.709838 & 2.249317 & -1.609521 \\ \mathrm{H} & 0.945956 & 2.543706 & 1.194478 \\ \mathrm{H} & 2.633232 & -1.817725 & 0.587890 \\ \mathrm{H} & 2.924506 & -0.085823 & 0.357665 \\ \mathrm{H} & 1.102409 & -1.148459 & 2.251316 \\ \mathrm{O} & 1.635231 & -0.873787 & -1.728271 \\ \mathrm{H} & 2.044163 & -1.747578 & -1.820112\end{array}$

d) $\mathrm{B}_{6}(\mathrm{C} 4) \cdot \cdot \mathrm{OH}$

Energies: $\mathrm{G}_{\mathrm{gas}}=-667.527979 \quad \mathrm{G}_{\mathrm{aq}}=-667.757960$

$\underline{\text { Coordinates }}$

\begin{tabular}{lrrr} 
& \multicolumn{1}{c}{$\mathrm{x}$} & $\mathrm{y}$ & $\mathrm{z}$ \\
$\mathrm{N}$ & 1.188755 & 1.895077 & 0.059420 \\
$\mathrm{C}$ & -0.134980 & 1.833989 & -0.287028 \\
$\mathrm{C}$ & -0.864403 & 0.702105 & -0.518647 \\
$\mathrm{C}$ & -0.201876 & -0.646629 & -0.413133 \\
$\mathrm{C}$ & 1.263300 & -0.483272 & -0.116409 \\
$\mathrm{C}$ & 1.873218 & 0.745312 & 0.149160 \\
$\mathrm{C}$ & 3.336785 & 0.813032 & 0.499372 \\
$\mathrm{O}$ & 2.026778 & -1.600334 & -0.138460 \\
$\mathrm{C}$ & -0.920079 & -1.577353 & 0.623888 \\
$\mathrm{O}$ & -1.099251 & -0.982003 & 1.884733 \\
$\mathrm{C}$ & -2.350062 & 0.774448 & -0.740742 \\
$\mathrm{O}$ & -3.090923 & 0.668131 & 0.506165 \\
$\mathrm{H}$ & -0.619670 & 2.806960 & -0.374316 \\
$\mathrm{H}$ & 3.547492 & 0.266859 & 1.425853 \\
$\mathrm{H}$ & 3.954742 & 0.360640 & -0.284364 \\
$\mathrm{H}$ & 3.623465 & 1.858276 & 0.626360 \\
$\mathrm{H}$ & 1.521382 & -2.313113 & -0.563999
\end{tabular}




$\begin{array}{lrrr}\mathrm{H} & -1.872704 & -1.878403 & 0.170444 \\ \mathrm{H} & -0.318707 & -2.479840 & 0.770624 \\ \mathrm{H} & -1.854389 & -0.376310 & 1.796792 \\ \mathrm{H} & -2.618418 & 1.709419 & -1.246380 \\ \mathrm{H} & -2.705205 & -0.058361 & -1.351815 \\ \mathrm{H} & -3.056262 & 1.527241 & 0.948483 \\ \mathrm{O} & -0.328408 & -1.431521 & -1.644005 \\ \mathrm{H} & 0.000000 & -0.892880 & -2.379841\end{array}$

e) $\mathrm{B}_{6}(\mathrm{C} 5)-\cdot \mathrm{OH}$

Energies: $\mathrm{G}_{\mathrm{gas}}=-667.533320 \quad \mathrm{G}_{\mathrm{aq}}=-667.754381$

Coordinates

\begin{tabular}{lrrr} 
& \multicolumn{1}{c}{$\mathrm{x}$} & $\mathrm{y}$ & $\mathrm{z}$ \\
$\mathrm{N}$ & 1.338387 & 1.899557 & 0.122746 \\
$\mathrm{C}$ & -0.004152 & 2.049175 & -0.055197 \\
$\mathrm{C}$ & -0.895520 & 0.991519 & -0.349227 \\
$\mathrm{C}$ & -0.405155 & -0.294224 & -0.457898 \\
$\mathrm{C}$ & 1.031863 & -0.570929 & -0.091229 \\
$\mathrm{C}$ & 1.857953 & 0.701257 & 0.101439 \\
$\mathrm{C}$ & 3.336252 & 0.543549 & 0.294029 \\
$\mathrm{O}$ & 1.100941 & -1.278371 & 1.151204 \\
$\mathrm{C}$ & -1.271712 & -1.504260 & -0.684977 \\
$\mathrm{O}$ & -1.681455 & -2.063330 & 0.585500 \\
$\mathrm{C}$ & -2.377449 & 1.277589 & -0.461330 \\
$\mathrm{O}$ & -3.125867 & 0.641630 & 0.596698 \\
$\mathrm{H}$ & -0.376035 & 3.068614 & 0.021688 \\
$\mathrm{H}$ & 3.550717 & -0.208358 & 1.062939 \\
$\mathrm{H}$ & 3.817079 & 0.212829 & -0.635363 \\
$\mathrm{H}$ & 3.768746 & 1.500744 & 0.589689 \\
$\mathrm{H}$ & 0.273272 & -1.783773 & 1.265072 \\
$\mathrm{H}$ & -2.150511 & -1.269769 & -1.293827 \\
$\mathrm{H}$ & -0.702915 & -2.288313 & -1.186493 \\
$\mathrm{H}$ & -2.323356 & -1.434905 & 0.956632 \\
$\mathrm{H}$ & -2.558538 & 2.359262 & -0.457467 \\
$\mathrm{H}$ & -2.801517 & 0.876716 & -1.385236 \\
$\mathrm{H}$ & -2.920266 & 1.093637 & 1.426645 \\
$\mathrm{O}$ & 1.595865 & -1.385252 & -1.118952 \\
$\mathrm{H}$ & 2.306268 & -1.903053 & -0.712769
\end{tabular}

f) $\mathrm{B}_{6}(\mathrm{C} 6) \cdot \cdot \mathrm{OH}$

Energies: $\mathrm{G}_{\mathrm{gas}}=-667.532466 \quad \mathrm{G}_{\mathrm{aq}}=-667.762229$

$\underline{\text { Coordinates }}$ 


$\begin{array}{lrrr} & \mathrm{x} & \mathrm{y} & \mathrm{z} \\ \mathrm{N} & -1.300456 & -1.762102 & -0.032016 \\ \mathrm{C} & -0.021864 & -1.958843 & -0.170310 \\ \mathrm{C} & 0.988895 & -0.952134 & -0.280429 \\ \mathrm{C} & 0.585045 & 0.409232 & -0.246331 \\ \mathrm{C} & -0.762020 & 0.686118 & -0.085075 \\ \mathrm{C} & -1.814517 & -0.403263 & 0.029548 \\ \mathrm{C} & -2.628009 & -0.245231 & 1.326278 \\ \mathrm{O} & -1.255347 & 1.931511 & 0.000000 \\ \mathrm{C} & 1.575869 & 1.540983 & -0.454945 \\ \mathrm{O} & 1.281330 & 2.700618 & 0.358402 \\ \mathrm{C} & 2.428514 & -1.367974 & -0.430028 \\ \mathrm{O} & 3.250961 & -0.975900 & 0.686066 \\ \mathrm{H} & 0.296656 & -3.002906 & -0.225591 \\ \mathrm{H} & -1.983172 & -0.331245 & 2.207716 \\ \mathrm{H} & -3.122966 & 0.728358 & 1.332790 \\ \mathrm{H} & -3.377198 & -1.040281 & 1.364638 \\ \mathrm{H} & -0.505962 & 2.558926 & 0.099208 \\ \mathrm{H} & 2.597635 & 1.207705 & -0.264126 \\ \mathrm{H} & 1.519031 & 1.911795 & -1.484640 \\ \mathrm{H} & 1.540409 & 2.513568 & 1.271299 \\ \mathrm{H} & 2.484593 & -2.453958 & -0.582240 \\ \mathrm{H} & 2.903171 & -0.896521 & -1.296807 \\ \mathrm{H} & 2.894451 & -1.388908 & 1.484458 \\ \mathrm{O} & -2.684769 & -0.231412 & -1.105154 \\ \mathrm{H} & -2.992328 & -1.123689 & -1.329300\end{array}$

\section{$2 . \cdot 00 H$ addition}

\subsection{REACTANTS}

a) Pyridoxine

Energies: $\mathrm{G}_{\mathrm{gas}}=-591.770767 \quad \mathrm{G}_{\mathrm{aq}}=-591.967495$

$\underline{\text { Coordinates }}$

$\begin{array}{lrrr} & \mathrm{x} & \mathrm{y} & \mathrm{z} \\ \mathrm{C} & 0.010624 & 2.001714 & 0.073482 \\ \mathrm{C} & -1.859630 & 0.662745 & -0.067344 \\ \mathrm{C} & -1.060173 & -0.492804 & 0.093001 \\ \mathrm{C} & 0.325908 & -0.373582 & 0.268084 \\ \mathrm{C} & 0.880258 & 0.923474 & 0.247645 \\ \mathrm{C} & -3.349532 & 0.537076 & -0.231889 \\ \mathrm{C} & 1.159865 & -1.613381 & 0.525282 \\ \mathrm{C} & 2.365388 & 1.175548 & 0.387882 \\ \mathrm{O} & 0.780951 & -2.721212 & -0.321003\end{array}$




$\begin{array}{rrrr}\mathrm{O} & -1.697245 & -1.697936 & 0.088458 \\ \mathrm{O} & 3.133284 & 0.631117 & -0.697116 \\ \mathrm{~N} & -1.316995 & 1.883493 & -0.072135 \\ \mathrm{H} & 0.402989 & 3.017553 & 0.053538 \\ \mathrm{H} & -3.599145 & -0.088961 & -1.096098 \\ \mathrm{H} & -3.804398 & 0.056403 & 0.642059 \\ \mathrm{H} & -3.781910 & 1.530398 & -0.363276 \\ \mathrm{H} & -1.030495 & -2.398743 & -0.054541 \\ \mathrm{H} & 2.224816 & -1.405823 & 0.409043 \\ \mathrm{H} & 0.986237 & -1.978769 & 1.544025 \\ \mathrm{H} & 1.091175 & -2.544588 & -1.219873 \\ \mathrm{H} & 2.543280 & 2.254822 & 0.482327 \\ \mathrm{H} & 2.775640 & 0.702345 & 1.285015 \\ \mathrm{H} & 2.838618 & 1.050425 & -1.516841\end{array}$

b) $\cdot \mathrm{OOH}$ Radical

Energies: $\mathrm{G}_{\mathrm{gas}}=-150.921387 \quad \mathrm{G}_{\mathrm{aq}}=-150.988356$

$\underline{\text { Coordinates }}$

$\begin{array}{cccc} & \mathrm{x} & \mathrm{y} & \mathrm{z} \\ \mathrm{O} & 0.055545 & 0.721264 & 0.000000 \\ \mathrm{O} & 0.055545 & -0.612053 & 0.000000 \\ \mathrm{H} & -0.888716 & -0.873690 & 0.000000\end{array}$

\subsection{TRANSITION STATES}

a) $\mathrm{B}_{6}(\mathrm{C} 2) \cdot \cdot \mathrm{OOH}$

Energies: $\mathrm{G}_{\mathrm{gas}}=-742.654071 \quad \mathrm{G}_{\mathrm{aq}}=-742.911585$

Coordinates

$\begin{array}{lrrr} & \mathrm{x} & \mathrm{y} & \mathrm{z} \\ \mathrm{C} & 0.672023 & 1.268102 & -0.069575 \\ \mathrm{C} & -0.586196 & 1.729236 & 0.433580 \\ \mathrm{~N} & -1.545535 & 0.890974 & 0.769059 \\ \mathrm{C} & -1.354348 & -0.458106 & 0.552637 \\ \mathrm{C} & -0.071298 & -1.000596 & 0.187645 \\ \mathrm{C} & 0.933572 & -0.115162 & -0.192573 \\ \mathrm{C} & -0.819931 & 3.205961 & 0.599842 \\ \mathrm{O} & -2.274133 & -0.770792 & -1.011191 \\ \mathrm{O} & -3.651814 & -0.724561 & -0.722554 \\ \mathrm{C} & 0.085636 & -2.504620 & 0.141625 \\ \mathrm{O} & 1.156916 & -2.986453 & 0.971093 \\ \mathrm{C} & 2.251844 & -0.582940 & -0.779622\end{array}$




$\begin{array}{rrrr}\mathrm{O} & 3.372554 & 0.221212 & -0.347862 \\ \mathrm{O} & 1.583643 & 2.213186 & -0.397052 \\ \mathrm{H} & -2.020507 & -1.096201 & 1.128748 \\ \mathrm{H} & -0.056412 & 3.656873 & 1.243123 \\ \mathrm{H} & -0.759421 & 3.721496 & -0.365564 \\ \mathrm{H} & -1.807637 & 3.366199 & 1.034877 \\ \mathrm{H} & 2.458653 & 1.779837 & -0.496017 \\ \mathrm{H} & 2.434064 & -1.632796 & -0.547056 \\ \mathrm{H} & 2.232240 & -0.464951 & -1.868999 \\ \mathrm{H} & 3.608219 & -0.029200 & 0.555991 \\ \mathrm{H} & -0.863807 & -2.978175 & 0.416257 \\ \mathrm{H} & 0.330465 & -2.848838 & -0.867808 \\ \mathrm{H} & 0.930103 & -2.815025 & 1.894829 \\ \mathrm{H} & -3.832354 & 0.231974 & -0.692615\end{array}$

b) $\mathrm{B}_{6}(\mathrm{C} 3)-\cdot \mathrm{OOH}$

Energies: $\mathrm{G}_{\mathrm{gas}}=-742.649888 \quad \mathrm{G}_{\mathrm{aq}}=-742.905914$

$\underline{\text { Coordinates }}$

\begin{tabular}{lrrr} 
& \multicolumn{1}{c}{$\mathrm{x}$} & $\mathrm{y}$ & $\mathrm{z}$ \\
$\mathrm{C}$ & -1.443195 & 0.138197 & -0.170587 \\
$\mathrm{C}$ & -1.705670 & -1.192522 & 0.286671 \\
$\mathrm{~N}$ & -0.714892 & -1.976463 & 0.747759 \\
$\mathrm{C}$ & 0.523867 & -1.508200 & 0.744973 \\
$\mathrm{C}$ & 0.932318 & -0.233205 & 0.190394 \\
$\mathrm{C}$ & -0.155295 & 0.643599 & -0.198782 \\
$\mathrm{C}$ & -3.103520 & -1.731003 & 0.286893 \\
$\mathrm{C}$ & 2.214667 & 0.375990 & 0.752021 \\
$\mathrm{O}$ & 1.958762 & 1.178348 & 1.908498 \\
$\mathrm{O}$ & 1.530122 & -0.715899 & -1.452463 \\
$\mathrm{O}$ & 2.692547 & -1.536393 & -1.394905 \\
$\mathrm{C}$ & 0.099886 & 2.033973 & -0.733709 \\
$\mathrm{O}$ & -0.875674 & 2.990222 & -0.263252 \\
$\mathrm{O}$ & -2.530174 & 0.864403 & -0.545297 \\
$\mathrm{H}$ & 1.295940 & -2.171812 & 1.132720 \\
$\mathrm{H}$ & -3.769663 & -1.094635 & 0.881713 \\
$\mathrm{H}$ & -3.520091 & -1.744599 & -0.727733 \\
$\mathrm{H}$ & -3.102523 & -2.743639 & 0.692951 \\
$\mathrm{H}$ & -2.271679 & 1.808334 & -0.580353 \\
$\mathrm{H}$ & 1.106265 & 2.380951 & -0.493907 \\
$\mathrm{H}$ & -0.004408 & 2.044803 & -1.824610 \\
$\mathrm{H}$ & -0.717288 & 3.146423 & 0.678711 \\
$\mathrm{H}$ & 2.930007 & -0.424209 & 0.970165 \\
$\mathrm{H}$ & 2.678754 & 1.038397 & 0.021453 \\
$\mathrm{H}$ & 1.664323 & 0.607786 & 2.631005 \\
$\mathrm{H}$ & 2.331594 & -2.418970 & -1.584310
\end{tabular}


c) $\mathrm{B}_{6}(\mathrm{C} 4)-\cdot \mathrm{OOH}$

Energies: $\mathrm{G}_{\mathrm{gas}}=-742.647841 \quad \mathrm{G}_{\mathrm{aq}}=-742.905415$

$\underline{\text { Coordinates }}$

$\begin{array}{lrrr} & \mathrm{x} & \mathrm{y} & \mathrm{z} \\ \mathrm{C} & -1.109968 & -0.472103 & -0.404555 \\ \mathrm{C} & -1.854649 & 0.722044 & -0.515049 \\ \mathrm{~N} & -1.313333 & 1.902787 & -0.221786 \\ \mathrm{C} & -0.007615 & 1.942873 & 0.158250 \\ \mathrm{C} & 0.823159 & 0.850987 & 0.259580 \\ \mathrm{C} & 0.262450 & -0.476740 & 0.053400 \\ \mathrm{C} & -3.291677 & 0.678645 & -0.957513 \\ \mathrm{C} & 2.283594 & 1.035609 & 0.575900 \\ \mathrm{O} & 3.127550 & 0.879785 & -0.591780 \\ \mathrm{C} & 1.203741 & -1.597500 & -0.417445 \\ \mathrm{O} & 1.727583 & -1.360046 & -1.707890 \\ \mathrm{O} & 0.112010 & -1.205251 & 1.722152 \\ \mathrm{O} & -0.892798 & -0.581130 & 2.487169 \\ \mathrm{O} & -1.768104 & -1.620652 & -0.667596 \\ \mathrm{H} & 0.379104 & 2.939295 & 0.369593 \\ \mathrm{H} & -3.390245 & 0.207189 & -1.941599 \\ \mathrm{H} & -3.896570 & 0.087542 & -0.260464 \\ \mathrm{H} & -3.681976 & 1.696530 & -1.002283 \\ \mathrm{H} & -1.239471 & -2.385117 & -0.396741 \\ \mathrm{H} & 1.997499 & -1.721542 & 0.328900 \\ \mathrm{H} & 0.675586 & -2.554204 & -0.463555 \\ \mathrm{H} & 2.391098 & -0.653326 & -1.614410 \\ \mathrm{H} & 2.449170 & 2.024387 & 1.019929 \\ \mathrm{H} & 2.643940 & 0.284627 & 1.283036 \\ \mathrm{H} & 2.970015 & 1.631529 & -1.180138 \\ \mathrm{H} & -0.408954 & 0.119046 & 2.958396\end{array}$

d) $\mathrm{B}_{6}(\mathrm{C} 5)-\cdot \mathrm{OOH}$

Energies: $\mathrm{G}_{\mathrm{gas}}=-742.648345 \quad \mathrm{G}_{\mathrm{aq}}=-742.906645$

$\underline{\text { Coordinates }}$

$\begin{array}{cccc} & & & \\ \mathrm{C} & 0.797891 & 0.256187 & -0.283749 \\ \mathrm{C} & 1.338668 & -1.081236 & -0.553900 \\ \mathrm{~N} & 0.647590 & -2.177429 & -0.294814 \\ \mathrm{C} & -0.623598 & -2.055848 & 0.138240 \\ \mathrm{C} & -1.306600 & -0.829617 & 0.297787\end{array}$




$\begin{array}{rrrr}\mathrm{C} & -0.624094 & 0.350619 & 0.036375 \\ \mathrm{C} & 2.718641 & -1.200484 & -1.119265 \\ \mathrm{C} & -2.767028 & -0.854927 & 0.706568 \\ \mathrm{O} & -3.642826 & -0.332197 & -0.301482 \\ \mathrm{C} & -1.211699 & 1.723129 & 0.254671 \\ \mathrm{O} & -0.919628 & 2.631360 & -0.832588 \\ \mathrm{O} & 1.411708 & 1.248201 & -0.974321 \\ \mathrm{O} & 1.361078 & 0.477817 & 1.433436 \\ \mathrm{O} & 2.736662 & 0.818993 & 1.474466 \\ \mathrm{H} & -1.143939 & -2.990037 & 0.341537 \\ \mathrm{H} & 2.745738 & -0.805353 & -2.142405 \\ \mathrm{H} & 3.431027 & -0.594276 & -0.547878 \\ \mathrm{H} & 3.022481 & -2.248501 & -1.125963 \\ \mathrm{H} & 0.831214 & 2.035970 & -0.987942 \\ \mathrm{H} & -2.289166 & 1.675112 & 0.420089 \\ \mathrm{H} & -0.734467 & 2.179385 & 1.129094 \\ \mathrm{H} & -1.462988 & 2.386087 & -1.594513 \\ \mathrm{H} & -3.053090 & -1.880782 & 0.973232 \\ \mathrm{H} & -2.945248 & -0.231691 & 1.587124 \\ \mathrm{H} & -3.581592 & -0.895773 & -1.084481 \\ \mathrm{H} & 3.137848 & 0.011525 & 1.839335\end{array}$

e) $\mathrm{B}_{6}(\mathrm{C} 6) \cdot \cdot \mathrm{OOH}$

Energies: $\mathrm{G}_{\mathrm{gas}}=-742.646574 \quad \mathrm{G}_{\mathrm{aq}}=-742.907821$

Coordinates

\begin{tabular}{lrrr} 
& \multicolumn{1}{c}{$\mathrm{x}$} & $\mathrm{y}$ & $\mathrm{z}$ \\
$\mathrm{C}$ & -0.398266 & 0.817784 & 0.209952 \\
$\mathrm{C}$ & -1.455049 & -0.127423 & 0.550902 \\
$\mathrm{~N}$ & -1.110250 & -1.446517 & 0.796126 \\
$\mathrm{C}$ & 0.089926 & -1.852183 & 0.447094 \\
$\mathrm{C}$ & 1.113992 & -1.023663 & -0.082347 \\
$\mathrm{C}$ & 0.862150 & 0.359465 & -0.182747 \\
$\mathrm{C}$ & -2.637154 & 0.369324 & 1.345502 \\
$\mathrm{O}$ & -2.125424 & -0.212754 & -1.160151 \\
$\mathrm{O}$ & -3.406699 & -0.793422 & -1.193036 \\
$\mathrm{C}$ & 2.439550 & -1.640299 & -0.461973 \\
$\mathrm{O}$ & 3.529980 & -1.185757 & 0.358200 \\
$\mathrm{C}$ & 1.882583 & 1.337351 & -0.733433 \\
$\mathrm{O}$ & 1.909489 & 2.582908 & 0.001806 \\
$\mathrm{O}$ & -0.725742 & 2.124776 & 0.234735 \\
$\mathrm{H}$ & 0.301081 & -2.912213 & 0.595643 \\
$\mathrm{H}$ & -2.343519 & 0.501111 & 2.393078 \\
$\mathrm{H}$ & -2.999668 & 1.327773 & 0.968967 \\
$\mathrm{H}$ & -3.425859 & -0.384581 & 1.308421
\end{tabular}




$\begin{array}{rrrr}\mathrm{H} & 0.095550 & 2.652923 & 0.134460 \\ \mathrm{H} & 2.880974 & 0.897985 & -0.754312 \\ \mathrm{H} & 1.607711 & 1.629788 & -1.753113 \\ \mathrm{H} & 2.361465 & 2.436940 & 0.844419 \\ \mathrm{H} & 2.358884 & -2.734201 & -0.422672 \\ \mathrm{H} & 2.729247 & -1.375306 & -1.483422 \\ \mathrm{H} & 3.362491 & -1.463246 & 1.269043 \\ \mathrm{H} & -3.995828 & -0.019499 & -1.163529\end{array}$

\subsection{PRODUCTS}

a) $\mathrm{B}_{6}(\mathrm{C} 2) \cdot \cdot \mathrm{OOH}$

Energies: $\mathrm{G}_{\mathrm{gas}}=-742.665244 \quad \mathrm{G}_{\mathrm{aq}}=-742.920021$

$\underline{\text { Coordinates }}$

\begin{tabular}{lrrr}
\multicolumn{1}{c}{$\mathrm{x}$} & $\mathrm{y}$ & $\mathrm{z}$ \\
$\mathrm{C}$ & -0.506922 & 1.801665 & 0.267934 \\
$\mathrm{~N}$ & -1.558839 & 1.061228 & 0.465117 \\
$\mathrm{C}$ & -1.467196 & -0.372987 & 0.326373 \\
$\mathrm{C}$ & -0.131872 & -0.984153 & 0.000000 \\
$\mathrm{C}$ & 0.949418 & -0.160033 & -0.225433 \\
$\mathrm{C}$ & 0.782312 & 1.242835 & -0.064470 \\
$\mathrm{O}$ & -2.341393 & -0.776163 & -0.760515 \\
$\mathrm{C}$ & -0.068027 & -2.494819 & -0.052878 \\
$\mathrm{O}$ & 0.910931 & -3.054903 & 0.844670 \\
$\mathrm{C}$ & 2.293052 & -0.691272 & -0.691425 \\
$\mathrm{O}$ & 3.405578 & -0.028639 & -0.049707 \\
$\mathrm{O}$ & 1.803263 & 2.118420 & -0.227180 \\
$\mathrm{C}$ & -0.637968 & 3.298349 & 0.379362 \\
$\mathrm{H}$ & -1.889463 & -0.829082 & 1.237466 \\
$\mathrm{H}$ & 0.042585 & 3.693471 & 1.141370 \\
$\mathrm{H}$ & -0.368204 & 3.784450 & -0.564697 \\
$\mathrm{H}$ & -1.666763 & 3.550678 & 0.640229 \\
$\mathrm{H}$ & 2.646678 & 1.617094 & -0.218481 \\
$\mathrm{H}$ & 2.364237 & -1.768898 & -0.543179 \\
$\mathrm{H}$ & 2.422130 & -0.473829 & -1.757603 \\
$\mathrm{H}$ & 3.493198 & -0.368107 & 0.851749 \\
$\mathrm{H}$ & -1.061641 & -2.908420 & 0.151949 \\
$\mathrm{H}$ & 0.224236 & -2.852626 & -1.045064 \\
$\mathrm{H}$ & 0.619523 & -2.896823 & 1.752366 \\
$\mathrm{O}$ & -3.711414 & -0.539892 & -0.336443 \\
$\mathrm{H}$ & -3.727139 & 0.435402 & -0.305301
\end{tabular}

b) $\mathrm{B}_{6}(\mathrm{C} 3) \cdot \cdot \mathrm{OOH}$ 
Energies: $\mathrm{G}_{\mathrm{gas}}=-742.657791 \quad \mathrm{G}_{\mathrm{aq}}=-742.909627$

Coordinates

\begin{tabular}{|c|c|c|c|}
\hline & & $\mathrm{y}$ & \\
\hline 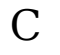 & 1.734611 & -1.188214 & 8986 \\
\hline & 0.760990 & -2.057130 & \\
\hline & 3165 & -1.675418 & -0. \\
\hline & -1.006373 & -0.341440 & 0.014985 \\
\hline & 0934 & 0.585478 & 0.504 \\
\hline & 8349 & 5067 & \\
\hline & -1.986909 & 0.298582 & -1.010845 \\
\hline & 9570 & 0.986286 & -2.055 \\
\hline & -1.733459 & -0.5 & \\
\hline & -0.177405 & 465 & 0.829 \\
\hline & 0.581529 & 0264 & 0.06 \\
\hline & 2.470407 & 5042 & 0.520 \\
\hline & 3.156527 & 2806 & -0.15 \\
\hline & -1.237468 & -2.4 & $-0.7 \subseteq$ \\
\hline & 3.757106 & -1.038008 & -0.834006 \\
\hline 7 & 3.627905 & -1.560428 & 0.834587 \\
\hline $\mathrm{H}$ & 3.191233 & -2.694852 & -0.473965 \\
\hline & 2.16 & 1.900737 & 0.4 \\
\hline $\mathrm{H}$ & -1.245255 & 2.224861 & 0.783451 \\
\hline $\mathrm{H}$ & 0.144072 & 1516 & 1.865279 \\
\hline & 0.299194 & 9702 & -0.859584 \\
\hline & -2.658594 & -0.467149 & -1.412611 \\
\hline 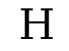 & -2.597789 & 1.047318 & -0.502688 \\
\hline & -0.864292 & 0.342742 & -2.63 \\
\hline & -2.967286 & -1.275346 & 1.038412 \\
\hline & -2.799053 & 0958 & 1.58343 \\
\hline
\end{tabular}

c) $\mathrm{B}_{6}(\mathrm{C} 4) \cdot \cdot \mathrm{OOH}$

Energies: $\mathrm{G}_{\mathrm{gas}}=-742.656169 \quad \mathrm{G}_{\mathrm{aq}}=-742.913008$

$\underline{\text { Coordinates }}$

$\begin{array}{lrrr} & \mathrm{x} & \mathrm{y} & \mathrm{z} \\ \mathrm{C} & -1.826555 & -0.933257 & -0.055440 \\ \mathrm{~N} & -1.131073 & -1.859965 & -0.725985 \\ \mathrm{C} & 0.182358 & -1.601227 & -1.015547 \\ \mathrm{C} & 0.889918 & -0.489322 & -0.657553 \\ \mathrm{C} & 0.205053 & 0.602325 & 0.115756 \\ \mathrm{C} & -1.244518 & 0.281652 & 0.321756 \\ \mathrm{C} & 2.369142 & -0.401135 & -0.915056 \\ \mathrm{O} & 3.156140 & -0.896008 & 0.203594 \\ \mathrm{C} & 0.945939 & 0.952884 & 1.462692\end{array}$




$\begin{array}{lrrr}\mathrm{O} & 1.239648 & -0.180511 & 2.237546 \\ \mathrm{O} & 0.305359 & 1.916614 & -0.551933 \\ \mathrm{O} & -2.025352 & 1.232589 & 0.877277 \\ \mathrm{C} & -3.278171 & -1.204288 & 0.238788 \\ \mathrm{H} & 0.678704 & -2.389340 & -1.583072 \\ \mathrm{H} & -3.477889 & -1.171738 & 1.315714 \\ \mathrm{H} & -3.921622 & -0.450082 & -0.228137 \\ \mathrm{H} & -3.540452 & -2.190801 & -0.146740 \\ \mathrm{H} & -1.574300 & 2.091008 & 0.818972 \\ \mathrm{H} & 1.850583 & 1.517141 & 1.200327 \\ \mathrm{H} & 0.304316 & 1.602534 & 2.063906 \\ \mathrm{H} & 2.006339 & -0.611150 & 1.820347 \\ \mathrm{H} & 2.633809 & -0.960277 & -1.820187 \\ \mathrm{H} & 2.695945 & 0.633622 & -1.046440 \\ \mathrm{H} & 3.148357 & -1.862586 & 0.171717 \\ \mathrm{O} & -0.489177 & 1.918877 & -1.769469 \\ \mathrm{H} & 0.161789 & 1.633140 & -2.433008\end{array}$

d) $\mathrm{B}_{6}(\mathrm{C} 5) \cdot \cdot \mathrm{OOH}$

Energies: $\mathrm{G}_{\mathrm{gas}}=-742.657221 \quad \mathrm{G}_{\mathrm{aq}}=-742.910182$

$\underline{\text { Coordinates }}$

\begin{tabular}{lrrr} 
& \multicolumn{1}{c}{$\mathrm{x}$} & $\mathrm{y}$ & $\mathrm{z}$ \\
$\mathrm{C}$ & -1.441454 & 1.110492 & -0.308012 \\
$\mathrm{~N}$ & -0.729505 & 2.192671 & -0.114103 \\
$\mathrm{C}$ & 0.573875 & 2.094150 & 0.257766 \\
$\mathrm{C}$ & 1.264864 & 0.872633 & 0.436799 \\
$\mathrm{C}$ & 0.570915 & -0.313199 & 0.327057 \\
$\mathrm{C}$ & -0.890695 & -0.293599 & -0.061148 \\
$\mathrm{C}$ & 2.763890 & 0.886669 & 0.650085 \\
$\mathrm{O}$ & 3.463035 & 0.274089 & -0.451422 \\
$\mathrm{C}$ & 1.207844 & -1.675360 & 0.441970 \\
$\mathrm{O}$ & 1.520274 & -2.200899 & -0.866615 \\
$\mathrm{O}$ & -1.145797 & -1.057026 & -1.212360 \\
$\mathrm{O}$ & -1.490275 & -0.907153 & 1.116250 \\
$\mathrm{C}$ & -2.834250 & 1.262440 & -0.839737 \\
$\mathrm{H}$ & 1.095985 & 3.038970 & 0.393162 \\
$\mathrm{H}$ & -3.019154 & 0.547462 & -1.646220 \\
$\mathrm{H}$ & -3.577921 & 1.049627 & -0.062928 \\
$\mathrm{H}$ & -2.971528 & 2.286100 & -1.193503 \\
$\mathrm{H}$ & -0.418194 & -1.690355 & -1.352777 \\
$\mathrm{H}$ & 2.108008 & -1.647972 & 1.062925 \\
$\mathrm{H}$ & 0.508399 & -2.387543 & 0.883496 \\
$\mathrm{H}$ & 2.251068 & -1.662578 & -1.210459 \\
$\mathrm{H}$ & 3.115867 & 1.914064 & 0.804078 \\
$\mathrm{H}$ & 3.055580 & 0.305084 & 1.528115
\end{tabular}


$\begin{array}{lrrr}\mathrm{H} & 3.401233 & 0.862785 & -1.216137 \\ \mathrm{O} & -2.903646 & -1.209479 & 0.876119 \\ \mathrm{H} & -3.281482 & -0.825952 & 1.684502\end{array}$

e) $\mathrm{B}_{6}(\mathrm{C} 6)-\cdot \mathrm{OOH}$

Energies: $\mathrm{G}_{\mathrm{gas}}=-742.655690 \quad \mathrm{G}_{\mathrm{aq}}=-742.916360$

$\underline{\text { Coordinates }}$

\begin{tabular}{lrrr} 
& \multicolumn{1}{c}{$\mathrm{x}$} & $\mathrm{y}$ & \multicolumn{1}{c}{$\mathrm{z}$} \\
$\mathrm{C}$ & 1.540093 & -0.151732 & -0.294874 \\
$\mathrm{~N}$ & 1.155461 & -1.538488 & -0.471593 \\
$\mathrm{C}$ & -0.067828 & -1.889933 & -0.218365 \\
$\mathrm{C}$ & -1.143557 & -1.026831 & 0.178878 \\
$\mathrm{C}$ & -0.889838 & 0.364847 & 0.247393 \\
$\mathrm{C}$ & 0.394605 & 0.808180 & -0.028539 \\
$\mathrm{C}$ & -2.497954 & -1.622409 & 0.462288 \\
$\mathrm{O}$ & -3.503727 & -1.226140 & -0.490856 \\
$\mathrm{C}$ & -1.962957 & 1.361744 & 0.648528 \\
$\mathrm{O}$ & -1.900124 & 2.585637 & -0.121214 \\
$\mathrm{O}$ & 0.732072 & 2.110021 & -0.073017 \\
$\mathrm{C}$ & 2.397622 & 0.287072 & -1.484529 \\
$\mathrm{O}$ & 2.274931 & -0.041951 & 0.968145 \\
$\mathrm{H}$ & -0.289963 & -2.955821 & -0.318995 \\
$\mathrm{H}$ & 1.791584 & 0.277160 & -2.395600 \\
$\mathrm{H}$ & 2.783637 & 1.297293 & -1.329832 \\
$\mathrm{H}$ & 3.216531 & -0.422593 & -1.606442 \\
$\mathrm{H}$ & -0.092803 & 2.644479 & -0.073823 \\
$\mathrm{H}$ & -2.958605 & 0.923372 & 0.563537 \\
$\mathrm{H}$ & -1.811422 & 1.685100 & 1.684388 \\
$\mathrm{H}$ & -2.258860 & 2.416694 & -1.003405 \\
$\mathrm{H}$ & -2.417960 & -2.716573 & 0.499927 \\
$\mathrm{H}$ & -2.892812 & -1.294750 & 1.429196 \\
$\mathrm{H}$ & -3.236978 & -1.544628 & -1.363999 \\
$\mathrm{O}$ & 3.559424 & -0.723119 & 0.868119 \\
$\mathrm{H}$ & 4.157697 & 0.038479 & 0.942099
\end{tabular}


Section 3.2. HYDROGEN/PROTON ABSTRACTION

$1 . \mathrm{B}_{6}+\cdot \mathrm{OH}----->\cdot \mathrm{B}_{6}+\mathrm{H}_{2} \mathrm{O}(1)$

\subsection{REACTANTS}

a) Pyridoxine

Energies: $\mathrm{G}_{\mathrm{gas}}=-591.770767 \quad \mathrm{G}_{\mathrm{aq}}=-591.967495$

$\underline{\text { Coordinates }}$

\begin{tabular}{lrrr} 
& \multicolumn{1}{c}{$\mathrm{x}$} & $\mathrm{y}$ & \multicolumn{1}{c}{$\mathrm{z}$} \\
$\mathrm{C}$ & 0.010624 & 2.001714 & 0.073482 \\
$\mathrm{C}$ & -1.859630 & 0.662745 & -0.067344 \\
$\mathrm{C}$ & -1.060173 & -0.492804 & 0.093001 \\
$\mathrm{C}$ & 0.325908 & -0.373582 & 0.268084 \\
$\mathrm{C}$ & 0.880258 & 0.923474 & 0.247645 \\
$\mathrm{C}$ & -3.349532 & 0.537076 & -0.231889 \\
$\mathrm{C}$ & 1.159865 & -1.613381 & 0.525282 \\
$\mathrm{C}$ & 2.365388 & 1.175548 & 0.387882 \\
$\mathrm{O}$ & 0.780951 & -2.721212 & -0.321003 \\
$\mathrm{O}$ & -1.697245 & -1.697936 & 0.088458 \\
$\mathrm{O}$ & 3.133284 & 0.631117 & -0.697116 \\
$\mathrm{~N}$ & -1.316995 & 1.883493 & -0.072135 \\
$\mathrm{H}$ & 0.402989 & 3.017553 & 0.053538 \\
$\mathrm{H}$ & -3.599145 & -0.088961 & -1.096098 \\
$\mathrm{H}$ & -3.804398 & 0.056403 & 0.642059 \\
$\mathrm{H}$ & -3.781910 & 1.530398 & -0.363276 \\
$\mathrm{H}$ & -1.030495 & -2.398743 & -0.054541 \\
$\mathrm{H}$ & 2.224816 & -1.405823 & 0.409043 \\
$\mathrm{H}$ & 0.986237 & -1.978769 & 1.544025 \\
$\mathrm{H}$ & 1.091175 & -2.544588 & -1.219873 \\
$\mathrm{H}$ & 2.543280 & 2.254822 & 0.482327 \\
$\mathrm{H}$ & 2.775640 & 0.702345 & 1.285015 \\
$\mathrm{H}$ & 2.838618 & 1.050425 & -1.516841
\end{tabular}

b) $\cdot \mathrm{OH}$ Radical

Energies: $\mathrm{G}_{\mathrm{gas}}=-75.746489 \quad \mathrm{G}_{\mathrm{aq}}=-75.784166$

$\underline{\text { Coordinates }}$
O $\quad 0.000000$
$\mathrm{y}$
$\mathrm{z}$
$\mathrm{H} \quad 0.000000$
$0.000000 \quad 0.108834$
$0.000000-0.870670$ 


\subsection{TRANSITION STATES}
a) $\mathrm{B}_{6}(\mathrm{C} 6)-\cdot \mathrm{OH}$

Energies: $\mathrm{G}_{\mathrm{gas}}=-667.510786 \quad \mathrm{G}_{\mathrm{aq}}=-667.739286$

$\underline{\text { Coordinates }}$

\begin{tabular}{lrrr} 
& \multicolumn{1}{c}{$\mathrm{x}$} & $\mathrm{y}$ & \multicolumn{1}{c}{$\mathrm{z}$} \\
$\mathrm{C}$ & 1.419745 & 0.347856 & -0.094484 \\
$\mathrm{C}$ & 1.023634 & 1.696170 & 0.038128 \\
$\mathrm{~N}$ & -0.281226 & 2.002082 & 0.010517 \\
$\mathrm{C}$ & -1.177069 & 1.061497 & -0.145866 \\
$\mathrm{C}$ & -0.904221 & -0.301949 & -0.288738 \\
$\mathrm{C}$ & 0.457707 & -0.663229 & -0.272689 \\
$\mathrm{C}$ & 2.035274 & 2.791839 & 0.212225 \\
$\mathrm{C}$ & -2.028339 & -1.304281 & -0.443831 \\
$\mathrm{O}$ & -2.106627 & -2.215299 & 0.663736 \\
$\mathrm{C}$ & 0.911856 & -2.095742 & -0.484637 \\
$\mathrm{O}$ & 1.989070 & -2.466473 & 0.401110 \\
$\mathrm{O}$ & 2.755594 & 0.086661 & -0.054725 \\
$\mathrm{O}$ & -3.652276 & 1.668747 & 0.066967 \\
$\mathrm{H}$ & -2.322817 & 1.378958 & -0.155386 \\
$\mathrm{H}$ & 2.680762 & 2.595943 & 1.075493 \\
$\mathrm{H}$ & 2.694118 & 2.860880 & -0.661122 \\
$\mathrm{H}$ & 1.521054 & 3.744642 & 0.348861 \\
$\mathrm{H}$ & 2.878833 & -0.866889 & 0.126844 \\
$\mathrm{H}$ & 0.081186 & -2.793768 & -0.372100 \\
$\mathrm{H}$ & 1.330750 & -2.210468 & -1.490879 \\
$\mathrm{H}$ & 1.629557 & -2.597048 & 1.289544 \\
$\mathrm{H}$ & -2.974970 & -0.771380 & -0.577589 \\
$\mathrm{H}$ & -1.877440 & -1.936220 & -1.324018 \\
$\mathrm{H}$ & -2.455977 & -1.733864 & 1.425639 \\
$\mathrm{H}$ & -3.534090 & 2.532580 & 0.503743
\end{tabular}

b) $\mathrm{B}_{6}(\mathrm{C} 7)-\cdot \mathrm{OH}$

Energies: $\mathrm{G}_{\mathrm{gas}}=-667.508661 \quad \mathrm{G}_{\mathrm{aq}}=-667.737816$

Coordinates

$\begin{array}{cccc} & \mathrm{x} & \mathrm{y} & \mathrm{z} \\ \mathrm{H} & -3.512035 & -1.638078 & 0.843706 \\ \mathrm{O} & -4.066574 & -0.939264 & 1.246006 \\ \mathrm{H} & -3.538791 & 0.116398 & 0.431532 \\ \mathrm{C} & -2.937050 & 0.650101 & -0.429166 \\ \mathrm{H} & -2.988213 & 1.721172 & -0.235323\end{array}$




$\begin{array}{lrrr}\mathrm{H} & -3.488081 & 0.375818 & -1.332791 \\ \mathrm{C} & -1.554520 & 0.098312 & -0.416035 \\ \mathrm{~N} & -1.425318 & -1.233868 & -0.511925 \\ \mathrm{C} & -0.203174 & -1.771182 & -0.491030 \\ \mathrm{H} & -0.155649 & -2.856977 & -0.560252 \\ \mathrm{C} & 0.977997 & -1.027024 & -0.381981 \\ \mathrm{C} & 2.311452 & -1.743473 & -0.352229 \\ \mathrm{H} & 2.156674 & -2.809303 & -0.564577 \\ \mathrm{H} & 2.992523 & -1.359685 & -1.117665 \\ \mathrm{O} & 3.014509 & -1.570970 & 0.886465 \\ \mathrm{H} & 2.489682 & -1.967101 & 1.595189 \\ \mathrm{C} & 0.867862 & 0.372025 & -0.283633 \\ \mathrm{C} & 2.079586 & 1.283225 & -0.230562 \\ \mathrm{H} & 2.985601 & 0.723175 & 0.005016 \\ \mathrm{H} & 2.217067 & 1.778148 & -1.198832 \\ \mathrm{O} & 1.914439 & 2.367601 & 0.707718 \\ \mathrm{H} & 2.001359 & 2.020216 & 1.606232 \\ \mathrm{C} & -0.421506 & 0.934436 & -0.285258 \\ \mathrm{O} & -0.624477 & 2.275872 & -0.193708 \\ \mathrm{H} & 0.190036 & 2.688869 & 0.158759\end{array}$

c) $\mathrm{B}_{6}(\mathrm{C} 8) \cdot \cdot \mathrm{OH}$ and $\mathrm{B}_{6}(\mathrm{O} 10) \cdot \cdot \cdot \mathrm{OH}$

Energies: $\mathrm{G}_{\mathrm{gas}}=-667.523720 \quad \mathrm{G}_{\mathrm{aq}}=-667.750202$

Coordinates

\begin{tabular}{lrrr} 
& \multicolumn{1}{c}{$\mathrm{x}$} & $\mathrm{y}$ & $\mathrm{z}$ \\
$\mathrm{C}$ & -1.174362 & 0.363106 & -0.173101 \\
$\mathrm{C}$ & -2.040045 & -0.788071 & -0.026664 \\
$\mathrm{~N}$ & -1.564532 & -2.011598 & 0.120914 \\
$\mathrm{C}$ & -0.222145 & -2.194548 & 0.107001 \\
$\mathrm{C}$ & 0.716658 & -1.194211 & -0.096085 \\
$\mathrm{C}$ & 0.246512 & 0.139003 & -0.299143 \\
$\mathrm{C}$ & -3.525875 & -0.575731 & 0.007703 \\
$\mathrm{C}$ & 2.170988 & -1.589972 & -0.175480 \\
$\mathrm{O}$ & 3.004114 & -0.901484 & 0.789758 \\
$\mathrm{C}$ & 1.064232 & 1.311087 & -0.624963 \\
$\mathrm{O}$ & 2.438243 & 1.269958 & -0.665064 \\
$\mathrm{O}$ & -1.713880 & 1.544075 & -0.163060 \\
$\mathrm{O}$ & -0.156794 & 3.167566 & 0.703681 \\
$\mathrm{H}$ & 0.108229 & -3.224036 & 0.233702 \\
$\mathrm{H}$ & -3.810403 & 0.049315 & 0.862225 \\
$\mathrm{H}$ & -3.866101 & -0.042503 & -0.886881 \\
$\mathrm{H}$ & -4.028488 & -1.541698 & 0.078526 \\
$\mathrm{H}$ & -0.989129 & 2.498638 & 0.323131 \\
$\mathrm{H}$ & 0.693887 & 1.871373 & -1.492846 \\
$\mathrm{H}$ & 0.664661 & 2.255832 & 0.207608
\end{tabular}




$\begin{array}{rrrr}\mathrm{H} & 2.788594 & 0.656110 & 0.019270 \\ \mathrm{H} & 2.268075 & -2.672264 & -0.038199 \\ \mathrm{H} & 2.611059 & -1.333299 & -1.142492 \\ \mathrm{H} & 2.670506 & -1.084572 & 1.679290 \\ \mathrm{H} & -0.148400 & 3.183387 & 1.672133\end{array}$

d) $\mathrm{B}_{6}(\mathrm{O} 11)-\cdot \mathrm{OH}$ and $\mathrm{B}_{6}(\mathrm{O} 12) \cdot \cdot \mathrm{OH}$

Energies: $\mathrm{G}_{\mathrm{gas}}=-667.513907 \quad \mathrm{G}_{\mathrm{aq}}=-667.737849$

Coordinates

\begin{tabular}{lrrr} 
& \multicolumn{1}{c}{$\mathrm{x}$} & $\mathrm{y}$ & $\mathrm{z}$ \\
$\mathrm{C}$ & 0.770239 & 1.980141 & 0.143665 \\
$\mathrm{~N}$ & 2.019345 & 1.560809 & 0.359871 \\
$\mathrm{C}$ & 2.304016 & 0.262510 & 0.189368 \\
$\mathrm{C}$ & 1.312375 & -0.658182 & -0.200041 \\
$\mathrm{C}$ & -0.007079 & -0.230950 & -0.424803 \\
$\mathrm{C}$ & -0.271352 & 1.136469 & -0.256590 \\
$\mathrm{C}$ & -1.079549 & -1.239441 & -0.817610 \\
$\mathrm{O}$ & -1.636431 & -1.968837 & 0.139097 \\
$\mathrm{O}$ & 1.583045 & -1.984766 & -0.380070 \\
$\mathrm{C}$ & 3.725821 & -0.181163 & 0.435911 \\
$\mathrm{C}$ & -1.638191 & 1.737563 & -0.539600 \\
$\mathrm{O}$ & -2.678705 & 0.737484 & -0.602471 \\
$\mathrm{O}$ & -2.866867 & -0.394595 & 1.515836 \\
$\mathrm{H}$ & -3.714451 & -0.743282 & 1.820215 \\
$\mathrm{H}$ & -2.265788 & -1.203884 & 1.060616 \\
$\mathrm{H}$ & -0.878768 & -1.772990 & -1.756056 \\
$\mathrm{H}$ & -2.117829 & -0.218654 & -1.003143 \\
$\mathrm{H}$ & 0.590258 & 3.043503 & 0.297223 \\
$\mathrm{H}$ & -2.903249 & 0.320120 & 0.438753 \\
$\mathrm{H}$ & -1.643485 & 2.256036 & -1.504735 \\
$\mathrm{H}$ & -1.913498 & 2.461179 & 0.234114 \\
$\mathrm{H}$ & 3.792972 & -0.923270 & 1.243671 \\
$\mathrm{H}$ & 4.183523 & -0.618137 & -0.462432 \\
$\mathrm{H}$ & 4.320010 & 0.686247 & 0.727962 \\
$\mathrm{H}$ & 2.508876 & -2.168506 & -0.176224
\end{tabular}

\subsection{COMPLEXES}

a) $\mathrm{B}_{6}(\mathrm{C} 6)-\cdot \mathrm{OH}$

Energies: $\mathrm{G}_{\mathrm{gas}}=-667.530100 \quad \mathrm{G}_{\mathrm{aq}}=-667.753214$

Coordinates 


\begin{tabular}{lrrr} 
& \multicolumn{1}{c}{$\mathrm{x}$} & $\mathrm{y}$ & $\mathrm{z}$ \\
$\mathrm{C}$ & 1.461334 & 0.231757 & 0.092262 \\
$\mathrm{C}$ & 1.758807 & -1.133769 & 0.271350 \\
$\mathrm{~N}$ & 0.763935 & -1.956085 & 0.662111 \\
$\mathrm{C}$ & -0.422781 & -1.499814 & 0.859136 \\
$\mathrm{C}$ & -0.845811 & -0.176492 & 0.744173 \\
$\mathrm{C}$ & 0.161817 & 0.723882 & 0.337432 \\
$\mathrm{C}$ & 3.136160 & -1.682022 & 0.042518 \\
$\mathrm{C}$ & -2.287466 & 0.231094 & 0.979936 \\
$\mathrm{O}$ & -2.934173 & 0.669456 & -0.213465 \\
$\mathrm{C}$ & -0.108989 & 2.209761 & 0.186649 \\
$\mathrm{O}$ & 0.508784 & 2.760924 & -0.995647 \\
$\mathrm{O}$ & 2.482012 & 1.043122 & -0.306989 \\
$\mathrm{O}$ & -2.656359 & -1.885333 & -1.500520 \\
$\mathrm{H}$ & -1.948710 & -2.248960 & -0.945218 \\
$\mathrm{H}$ & 3.487882 & -1.448261 & -0.968434 \\
$\mathrm{H}$ & 3.858024 & -1.234952 & 0.735908 \\
$\mathrm{H}$ & 3.127539 & -2.764185 & 0.184718 \\
$\mathrm{H}$ & 2.095690 & 1.861682 & -0.678302 \\
$\mathrm{H}$ & -1.180544 & 2.413141 & 0.175054 \\
$\mathrm{H}$ & 0.344920 & 2.760657 & 1.018389 \\
$\mathrm{H}$ & 0.000000 & 2.472286 & -1.769291 \\
$\mathrm{H}$ & -2.819710 & -0.614825 & 1.433125 \\
$\mathrm{H}$ & -2.350672 & 1.068111 & 1.682773 \\
$\mathrm{H}$ & -2.999235 & -0.103383 & -0.804819 \\
$\mathrm{H}$ & -3.287727 & -2.600444 & -1.646442
\end{tabular}

b) $\mathrm{B}_{6}(\mathrm{C} 7) \cdot-\mathrm{OH}$

Energies: $\mathrm{G}_{\mathrm{gas}}=-667.553700 \quad \mathrm{G}_{\mathrm{aq}}=-667.783921$

Coordinates

$\begin{array}{lrrr} & \mathrm{x} & \mathrm{y} & \mathrm{z} \\ \mathrm{C} & 1.068804 & 1.662731 & 0.487889 \\ \mathrm{~N} & 0.071694 & 2.501696 & 0.065897 \\ \mathrm{C} & -1.061961 & 1.970909 & -0.364978 \\ \mathrm{C} & -1.332344 & 0.589215 & -0.442928 \\ \mathrm{C} & -0.318981 & -0.303590 & -0.033686 \\ \mathrm{C} & 0.873999 & 0.242062 & 0.442811 \\ \mathrm{C} & -2.677142 & 0.116008 & -0.941450 \\ \mathrm{O} & -3.440920 & -0.566186 & 0.067184 \\ \mathrm{C} & -0.448742 & -1.810804 & -0.138822 \\ \mathrm{O} & 0.049286 & -2.475260 & 1.047180 \\ \mathrm{O} & 1.910444 & -0.552427 & 0.849055 \\ \mathrm{C} & 2.263555 & 2.227213 & 0.957072 \\ \mathrm{H} & -1.828760 & 2.678163 & -0.682573\end{array}$




$\begin{array}{rrrr}\mathrm{H} & 3.071763 & 1.606403 & 1.321263 \\ \mathrm{H} & 2.366660 & 3.305588 & 0.961195 \\ \mathrm{H} & 1.535333 & -1.428750 & 1.090286 \\ \mathrm{H} & -1.480678 & -2.111960 & -0.324433 \\ \mathrm{H} & 0.187095 & -2.180479 & -0.950259 \\ \mathrm{H} & -0.585387 & -2.344308 & 1.765163 \\ \mathrm{H} & -3.240801 & 0.969698 & -1.340098 \\ \mathrm{H} & -2.575540 & -0.610761 & -1.752885 \\ \mathrm{H} & -3.612029 & 0.052898 & 0.789790 \\ \mathrm{H} & 3.400337 & -1.584073 & -2.276935 \\ \mathrm{O} & 2.642130 & -1.756845 & -1.707076 \\ \mathrm{H} & 2.769499 & -1.221007 & -0.907991\end{array}$

c) $\mathrm{B}_{6}(\mathrm{C} 8)-\cdot \mathrm{OH}$

Energies: $\mathrm{G}_{\mathrm{gas}}=-667.570661 \quad \mathrm{G}_{\mathrm{aq}}=-667.799611$

Coordinates

\begin{tabular}{lrrr} 
& \multicolumn{1}{c}{$\mathrm{x}$} & $\mathrm{y}$ & $\mathrm{z}$ \\
$\mathrm{N}$ & 2.142706 & -1.616730 & 0.072796 \\
$\mathrm{C}$ & 0.864297 & -1.993012 & 0.268884 \\
$\mathrm{C}$ & -0.216535 & -1.128650 & 0.345704 \\
$\mathrm{C}$ & 0.026869 & 0.284806 & 0.205773 \\
$\mathrm{C}$ & 1.389569 & 0.664548 & 0.000000 \\
$\mathrm{C}$ & 2.403655 & -0.308790 & -0.062060 \\
$\mathrm{C}$ & 3.832749 & 0.108845 & -0.283606 \\
$\mathrm{O}$ & 1.782331 & 1.960165 & -0.155945 \\
$\mathrm{C}$ & -1.039009 & 1.195560 & 0.268727 \\
$\mathrm{O}$ & -0.807888 & 2.545760 & 0.155639 \\
$\mathrm{C}$ & -1.594472 & -1.688012 & 0.574049 \\
$\mathrm{O}$ & -2.483086 & -1.537506 & -0.562418 \\
$\mathrm{H}$ & 0.701211 & -3.064614 & 0.378309 \\
$\mathrm{H}$ & 3.944811 & 0.649692 & -1.230377 \\
$\mathrm{H}$ & 4.173753 & 0.788028 & 0.506154 \\
$\mathrm{H}$ & 4.466060 & -0.779767 & -0.296304 \\
$\mathrm{H}$ & 1.010424 & 2.548545 & -0.106913 \\
$\mathrm{H}$ & -3.721505 & -0.123120 & -0.607884 \\
$\mathrm{H}$ & -2.073407 & 0.907286 & 0.382252 \\
$\mathrm{H}$ & -1.647304 & 3.015984 & 0.065605 \\
$\mathrm{H}$ & -1.519330 & -2.751068 & 0.831475 \\
$\mathrm{H}$ & -2.113805 & -1.181946 & 1.394052 \\
$\mathrm{H}$ & -2.013280 & -1.821970 & -1.358518 \\
$\mathrm{O}$ & -4.132162 & 0.746652 & -0.436171 \\
$\mathrm{H}$ & -5.082863 & 0.597725 & -0.380061
\end{tabular}

d) $\mathrm{B}_{6}(\mathrm{C} 9) \cdot \cdot \mathrm{OH}$ 
Energies: $\mathrm{G}_{\mathrm{gas}}=-667.561539 \quad \mathrm{G}_{\mathrm{aq}}=-667.796764$

$\underline{\text { Coordinates }}$

\begin{tabular}{lrrr} 
& \multicolumn{1}{c}{$\mathrm{x}$} & $\mathrm{y}$ & $\mathrm{z}$ \\
$\mathrm{C}$ & 0.000000 & -0.216187 & 0.265149 \\
$\mathrm{C}$ & 1.304300 & -0.589624 & -0.066939 \\
$\mathrm{C}$ & 2.301105 & 0.385249 & -0.331558 \\
$\mathrm{~N}$ & 2.023958 & 1.698101 & -0.275600 \\
$\mathrm{C}$ & 0.789641 & 2.079220 & 0.015099 \\
$\mathrm{C}$ & -0.295372 & 1.190182 & 0.284894 \\
$\mathrm{O}$ & 1.712513 & -1.884132 & -0.162746 \\
$\mathrm{C}$ & 3.700198 & -0.036610 & -0.679471 \\
$\mathrm{C}$ & -1.533800 & 1.815932 & 0.525482 \\
$\mathrm{O}$ & -2.729379 & 1.126646 & 0.596210 \\
$\mathrm{C}$ & -1.083120 & -1.239943 & 0.557205 \\
$\mathrm{O}$ & -0.496269 & -2.458192 & 1.075484 \\
$\mathrm{H}$ & -1.606467 & 2.897933 & 0.554278 \\
$\mathrm{H}$ & 0.609519 & 3.153131 & 0.038686 \\
$\mathrm{H}$ & 4.134804 & -0.659747 & 0.111008 \\
$\mathrm{H}$ & 3.718231 & -0.641517 & -1.594151 \\
$\mathrm{H}$ & 4.317328 & 0.852241 & -0.822950 \\
$\mathrm{H}$ & 1.016900 & -2.449626 & 0.231798 \\
$\mathrm{H}$ & -1.653633 & -1.467430 & -0.353338 \\
$\mathrm{H}$ & -1.780704 & -0.846381 & 1.298915 \\
$\mathrm{H}$ & -1.159892 & -3.158312 & 1.039550 \\
$\mathrm{H}$ & -3.415424 & 1.700916 & 0.960281 \\
$\mathrm{H}$ & -3.443947 & 0.024810 & -0.900885 \\
$\mathrm{O}$ & -3.758329 & -0.695867 & -1.470608 \\
$\mathrm{H}$ & -3.829334 & -0.329683 & -2.359875
\end{tabular}

e) $\mathrm{B}_{6}(\mathrm{O} 10) \cdot \cdot \mathrm{OH}$

Energies: $\mathrm{G}_{\mathrm{gas}}=-667.569780 \quad \mathrm{G}_{\mathrm{aq}}=-667.786530$

$\underline{\text { Coordinates }}$

$\begin{array}{crrr} & \mathrm{x} & \mathrm{y} & \mathrm{z} \\ \mathrm{N} & 1.796473 & -1.823132 & -0.356801 \\ \mathrm{C} & 0.500508 & -2.185914 & -0.277301 \\ \mathrm{C} & -0.560934 & -1.358498 & 0.160613 \\ \mathrm{C} & -0.262402 & -0.054564 & 0.534634 \\ \mathrm{C} & 1.105271 & 0.410876 & 0.362678 \\ \mathrm{C} & 2.119029 & -0.574764 & -0.051024 \\ \mathrm{C} & 3.547698 & -0.145255 & -0.160280 \\ \mathrm{O} & 1.437019 & 1.618391 & 0.521283 \\ \mathrm{C} & -1.322242 & 0.900289 & 1.044299\end{array}$




$\begin{array}{rrrr}\mathrm{O} & -2.213701 & 1.307835 & 0.000000 \\ \mathrm{C} & -1.983673 & -1.891214 & 0.151054 \\ \mathrm{O} & -2.753417 & -1.350119 & -0.912977 \\ \mathrm{H} & 0.285678 & -3.212764 & -0.567438 \\ \mathrm{H} & 3.657387 & 0.638816 & -0.918941 \\ \mathrm{H} & 3.896283 & 0.293029 & 0.781470 \\ \mathrm{H} & 4.167696 & -1.001869 & -0.427661 \\ \mathrm{H} & -1.786218 & 2.058270 & -0.461363 \\ \mathrm{H} & -1.935310 & 0.402086 & 1.801453 \\ \mathrm{H} & -0.850384 & 1.769011 & 1.509982 \\ \mathrm{H} & -1.969615 & -2.974265 & 0.000000 \\ \mathrm{H} & -2.464695 & -1.703979 & 1.123277 \\ \mathrm{H} & -2.778665 & -0.380288 & -0.803485 \\ \mathrm{H} & 0.342186 & 2.965002 & -0.246101 \\ \mathrm{O} & -0.450019 & 3.357955 & -0.662273 \\ \mathrm{H} & -0.158233 & 3.830643 & -1.449918\end{array}$

f) $\mathrm{B}_{6}(\mathrm{O} 11) \cdot \cdot \mathrm{OH}$

Energies: $\mathrm{G}_{\mathrm{gas}}=-667.534751 \quad \mathrm{G}_{\mathrm{aq}}=-667.751742$

$\underline{\text { Coordinates }}$

\begin{tabular}{lrrr} 
& \multicolumn{1}{c}{$\mathrm{x}$} & $\mathrm{y}$ & \multicolumn{1}{c}{$\mathrm{z}$} \\
$\mathrm{N}$ & 1.867961 & -1.663782 & -0.279848 \\
$\mathrm{C}$ & 0.606446 & -1.994592 & 0.043806 \\
$\mathrm{C}$ & -0.380116 & -1.088470 & 0.441164 \\
$\mathrm{C}$ & 0.022629 & 0.255213 & 0.559428 \\
$\mathrm{C}$ & 1.323721 & 0.617953 & 0.151092 \\
$\mathrm{C}$ & 2.247135 & -0.389664 & -0.231757 \\
$\mathrm{C}$ & 3.649467 & -0.026134 & -0.632925 \\
$\mathrm{O}$ & 1.747409 & 1.902064 & 0.127113 \\
$\mathrm{C}$ & -0.862860 & 1.382718 & 1.112721 \\
$\mathrm{O}$ & -0.966102 & 2.203377 & 0.000000 \\
$\mathrm{C}$ & -1.782082 & -1.605743 & 0.724132 \\
$\mathrm{O}$ & -2.596194 & -1.652407 & -0.433962 \\
$\mathrm{H}$ & 0.366156 & -3.054368 & 0.000000 \\
$\mathrm{H}$ & 3.648440 & 0.661764 & -1.485903 \\
$\mathrm{H}$ & 4.173926 & 0.484840 & 0.182558 \\
$\mathrm{H}$ & 4.191813 & -0.934263 & -0.900169 \\
$\mathrm{H}$ & 0.962381 & 2.480023 & 0.038132 \\
$\mathrm{H}$ & -1.832742 & 0.995460 & 1.442934 \\
$\mathrm{H}$ & -0.349365 & 1.881992 & 1.949029 \\
$\mathrm{H}$ & -2.840367 & -0.743822 & -0.704645 \\
$\mathrm{H}$ & -1.706073 & -2.636546 & 1.082842 \\
$\mathrm{H}$ & -2.258848 & -1.023686 & 1.525507 \\
$\mathrm{H}$ & -2.461401 & 1.600721 & -0.924431 \\
$\mathrm{O}$ & -3.231195 & 1.022058 & -1.101141
\end{tabular}


g) $\mathrm{B}_{6}(\mathrm{O} 12) \cdot \cdot \mathrm{OH}$

Energies: $\mathrm{G}_{\mathrm{gas}}=-667.540540 \quad \mathrm{G}_{\mathrm{aq}}=-667.766887$

Coordinates

$\begin{array}{lrrr} & & & \\ \mathrm{C} & -0.719862 & -0.996481 & -0.057129 \\ \mathrm{C} & -2.134020 & -1.027614 & -0.038274 \\ \mathrm{~N} & -2.857117 & 0.090683 & 0.066928 \\ \mathrm{C} & -2.214428 & 1.262258 & 0.159895 \\ \mathrm{C} & -0.825417 & 1.394053 & 0.151191 \\ \mathrm{C} & -0.039590 & 0.228836 & 0.036256 \\ \mathrm{C} & -2.858833 & -2.341463 & -0.147342 \\ \mathrm{C} & -0.228216 & 2.779672 & 0.265982 \\ \mathrm{O} & 0.715947 & 3.140386 & -0.661900 \\ \mathrm{C} & 1.471035 & 0.297222 & -0.053876 \\ \mathrm{O} & 2.054080 & -0.872040 & 0.542576 \\ \mathrm{O} & -0.080427 & -2.190933 & -0.184311 \\ \mathrm{H} & -2.844748 & 2.146610 & 0.242155 \\ \mathrm{H} & -2.621529 & -2.846813 & -1.090891 \\ \mathrm{H} & -2.558236 & -3.024586 & 0.655225 \\ \mathrm{H} & -3.934366 & -2.164071 & -0.093115 \\ \mathrm{H} & 0.863914 & -2.041738 & 0.040773 \\ \mathrm{H} & 1.850589 & 1.193229 & 0.448095 \\ \mathrm{H} & 1.769482 & 0.371826 & -1.109564 \\ \mathrm{H} & 3.004610 & -0.904990 & 0.322897 \\ \mathrm{H} & -1.021380 & 3.548854 & 0.312905 \\ \mathrm{H} & 0.297307 & 2.904833 & 1.241935 \\ \mathrm{H} & 5.447491 & -0.940626 & 0.620256 \\ \mathrm{O} & 4.798141 & -0.942535 & -0.094373 \\ \mathrm{H} & 5.140749 & -1.535235 & -0.775342\end{array}$

\subsection{PRODUCTS}
a) $\cdot \mathrm{B}_{6}(\mathrm{C} 6)$

Energies: $\mathrm{G}_{\mathrm{gas}}=-591.102531$

$$
\mathrm{G}_{\mathrm{aq}}=-591.298647
$$

\section{$\underline{\text { Coordinates }}$}
$\begin{array}{cc} & \mathrm{x} \\ \text { C } & 1.190982\end{array}$
$\mathrm{y} \quad \mathrm{z}$
$\begin{array}{llll}\text { C } & 1.460633 & -1.204672 & -0.229242\end{array}$ 


$\begin{array}{lrrr}\mathrm{N} & 0.412095 & -2.028123 & -0.433032 \\ \mathrm{C} & -0.792898 & -1.607334 & -0.311912 \\ \mathrm{C} & -1.197534 & -0.315635 & 0.024488 \\ \mathrm{C} & -0.140305 & 0.584545 & 0.266607 \\ \mathrm{C} & 2.860750 & -1.723610 & -0.373572 \\ \mathrm{C} & -2.670705 & 0.016854 & 0.164463 \\ \mathrm{O} & -3.511670 & -0.929812 & -0.470741 \\ \mathrm{C} & -0.386016 & 2.008297 & 0.726723 \\ \mathrm{O} & 0.473440 & 2.964132 & 0.073369 \\ \mathrm{O} & 2.265071 & 0.943608 & 0.340033 \\ \mathrm{H} & 3.428390 & -1.126325 & -1.095562 \\ \mathrm{H} & 3.403189 & -1.665304 & 0.577248 \\ \mathrm{H} & 2.831972 & -2.763218 & -0.704721 \\ \mathrm{H} & 1.966868 & 1.873281 & 0.303960 \\ \mathrm{H} & -1.433120 & 2.297060 & 0.597857 \\ \mathrm{H} & -0.146012 & 2.107982 & 1.791213 \\ \mathrm{H} & 0.218150 & 3.035478 & -0.857056 \\ \mathrm{H} & -2.930342 & 0.098931 & 1.233431 \\ \mathrm{H} & -2.898693 & 0.985093 & -0.296148 \\ \mathrm{H} & -3.089246 & -1.800840 & -0.412715\end{array}$

b) $\cdot \mathrm{B}_{6}(\mathrm{C} 7)$

Energies: $\mathrm{G}_{\mathrm{gas}}=-591.131656 \quad \mathrm{G}_{\mathrm{aq}}=-591.329448$

Coordinates

\begin{tabular}{lrrr} 
& $\mathrm{x}$ & $\mathrm{y}$ & \multicolumn{1}{c}{$\mathrm{z}$} \\
$\mathrm{N}$ & 1.343325 & -1.925135 & -0.041256 \\
$\mathrm{C}$ & 0.031623 & -2.011103 & 0.118317 \\
$\mathrm{C}$ & -0.837493 & -0.912741 & 0.272773 \\
$\mathrm{C}$ & -0.274591 & 0.383231 & 0.255621 \\
$\mathrm{C}$ & 1.106050 & 0.494615 & 0.068375 \\
$\mathrm{C}$ & 1.919757 & -0.683891 & -0.076370 \\
$\mathrm{C}$ & 3.306308 & -0.595612 & -0.253921 \\
$\mathrm{O}$ & 1.740913 & 1.692803 & 0.030339 \\
$\mathrm{C}$ & -1.105301 & 1.630285 & 0.491659 \\
$\mathrm{O}$ & -0.714622 & 2.728153 & -0.363215 \\
$\mathrm{C}$ & -2.320227 & -1.153158 & 0.431531 \\
$\mathrm{O}$ & -3.096488 & -0.631511 & -0.660610 \\
$\mathrm{H}$ & -0.382498 & -3.019676 & 0.132198 \\
$\mathrm{H}$ & 3.805936 & 0.363333 & -0.295972 \\
$\mathrm{H}$ & 3.880259 & -1.509341 & -0.350369 \\
$\mathrm{H}$ & 1.071101 & 2.393316 & -0.110988 \\
$\mathrm{H}$ & -2.169560 & 1.424875 & 0.366187 \\
$\mathrm{H}$ & -0.941352 & 2.006653 & 1.507925 \\
$\mathrm{H}$ & -1.024143 & 2.547393 & -1.261593 \\
$\mathrm{H}$ & -2.504160 & -2.228466 & 0.554692
\end{tabular}


$\mathrm{H} \quad-2.721666 \quad-0.654415 \quad 1.319163$

$\mathrm{H} \quad-2.812360 \quad-1.073051 \quad-1.472475$

c) $\cdot \mathrm{B}_{6}(\mathrm{C} 8)$

Energies: $\mathrm{G}_{\mathrm{gas}}=-591.144028 \quad \mathrm{G}_{\mathrm{aq}}=-591.345021$

$\underline{\text { Coordinates }}$

\begin{tabular}{lrrr} 
& \multicolumn{1}{c}{$\mathrm{x}$} & $\mathrm{y}$ & \multicolumn{1}{c}{$\mathrm{z}$} \\
$\mathrm{C}$ & 1.070991 & 0.491498 & 0.000000 \\
$\mathrm{C}$ & 1.836954 & -0.688778 & -0.037959 \\
$\mathrm{~N}$ & 1.275621 & -1.901801 & 0.042370 \\
$\mathrm{C}$ & -0.065036 & -1.977337 & 0.162739 \\
$\mathrm{C}$ & -0.920749 & -0.889490 & 0.209756 \\
$\mathrm{C}$ & -0.352842 & 0.430143 & 0.111531 \\
$\mathrm{C}$ & 3.334196 & -0.607794 & -0.172870 \\
$\mathrm{C}$ & -2.400337 & -1.107174 & 0.401947 \\
$\mathrm{O}$ & -3.215239 & -0.593569 & -0.665708 \\
$\mathrm{C}$ & -1.189497 & 1.559986 & 0.123853 \\
$\mathrm{O}$ & -0.640439 & 2.823499 & 0.137653 \\
$\mathrm{O}$ & 1.759630 & 1.662090 & -0.109712 \\
$\mathrm{H}$ & -0.474151 & -2.984014 & 0.236868 \\
$\mathrm{H}$ & 3.621669 & -0.096016 & -1.098592 \\
$\mathrm{H}$ & 3.775906 & -0.035509 & 0.650911 \\
$\mathrm{H}$ & 3.746468 & -1.618191 & -0.174740 \\
$\mathrm{H}$ & 1.145878 & 2.413267 & -0.065477 \\
$\mathrm{H}$ & -2.268367 & 1.489913 & 0.094630 \\
$\mathrm{H}$ & -1.321843 & 3.496248 & 0.019237 \\
$\mathrm{H}$ & -2.591734 & -2.178445 & 0.547513 \\
$\mathrm{H}$ & -2.761708 & -0.585711 & 1.295514 \\
$\mathrm{H}$ & -2.915160 & -0.991414 & -1.494219
\end{tabular}

d) $\cdot B_{6}(C 9)$

Energies: $\mathrm{G}_{\mathrm{gas}}=-591.139957 \quad \mathrm{G}_{\mathrm{aq}}=-591.344235$

Coordinates

$\begin{array}{cccc} & & & \\ \mathrm{C} & -1.171110 & 0.133971 & 0.074321 \\ \mathrm{C} & -1.388579 & -1.264379 & -0.011621 \\ \mathrm{~N} & -0.360966 & -2.123724 & -0.087273 \\ \mathrm{C} & 0.883554 & -1.656782 & -0.078434 \\ \mathrm{C} & 1.216000 & -0.273316 & 0.000000 \\ \mathrm{C} & 0.124245 & 0.655150 & 0.079385 \\ \mathrm{C} & -2.785932 & -1.817804 & -0.015758\end{array}$




$\begin{array}{lrrr}\mathrm{C} & 2.568934 & 0.120642 & 0.053124 \\ \mathrm{O} & 3.541630 & -0.839051 & -0.032143 \\ \mathrm{C} & 0.386217 & 2.134930 & 0.233865 \\ \mathrm{O} & -0.651257 & 2.899140 & -0.416667 \\ \mathrm{O} & -2.292031 & 0.908068 & 0.166969 \\ \mathrm{H} & 1.680588 & -2.390501 & -0.140538 \\ \mathrm{H} & -3.320692 & -1.561065 & 0.906882 \\ \mathrm{H} & -3.375852 & -1.402006 & -0.841157 \\ \mathrm{H} & -2.739160 & -2.903974 & -0.113362 \\ \mathrm{H} & -2.040739 & 1.833519 & -0.013772 \\ \mathrm{H} & 1.347679 & 2.402077 & -0.217134 \\ \mathrm{H} & 0.423388 & 2.403278 & 1.301748 \\ \mathrm{H} & -0.555348 & 3.830695 & -0.181192 \\ \mathrm{H} & 2.905820 & 1.144924 & 0.153764 \\ \mathrm{H} & 4.414370 & -0.430599 & 0.000000\end{array}$

e) $\cdot \mathrm{B}_{6}(\mathrm{O} 10)$

Energies: $\mathrm{G}_{\mathrm{gas}}=-591.140797 \quad \mathrm{G}_{\mathrm{aq}}=-591.334827$

$\underline{\text { Coordinates }}$

\begin{tabular}{lrrr} 
& \multicolumn{1}{c}{$\mathrm{x}$} & $\mathrm{y}$ & $\mathrm{z}$ \\
$\mathrm{C}$ & -0.166125 & -1.880899 & 0.000000 \\
$\mathrm{C}$ & 1.829320 & -0.751360 & -0.063629 \\
$\mathrm{C}$ & 1.137534 & 0.520425 & 0.184791 \\
$\mathrm{C}$ & -0.310929 & 0.493342 & 0.361642 \\
$\mathrm{C}$ & -0.956584 & -0.727450 & 0.259446 \\
$\mathrm{C}$ & 3.314181 & -0.749920 & -0.235920 \\
$\mathrm{C}$ & -0.971663 & 1.829527 & 0.632999 \\
$\mathrm{C}$ & -2.459578 & -0.914251 & 0.343037 \\
$\mathrm{O}$ & -0.743264 & 2.759247 & -0.420234 \\
$\mathrm{O}$ & 1.781418 & 1.601407 & 0.229966 \\
$\mathrm{O}$ & -3.057205 & -1.016712 & -0.950724 \\
$\mathrm{~N}$ & 1.168748 & -1.901499 & -0.148166 \\
$\mathrm{H}$ & -0.662487 & -2.845637 & -0.089088 \\
$\mathrm{H}$ & 3.602406 & -0.122546 & -1.087549 \\
$\mathrm{H}$ & 3.805552 & -0.312779 & 0.640844 \\
$\mathrm{H}$ & 3.667492 & -1.769941 & -0.392109 \\
$\mathrm{H}$ & 0.212836 & 2.925664 & -0.436931 \\
$\mathrm{H}$ & -2.054152 & 1.730650 & 0.721516 \\
$\mathrm{H}$ & -0.594988 & 2.222999 & 1.588839 \\
$\mathrm{H}$ & -2.687188 & -1.862904 & 0.836970 \\
$\mathrm{H}$ & -2.930773 & -0.120083 & 0.932781 \\
$\mathrm{H}$ & -2.884464 & -0.202953 & -1.444375
\end{tabular}

f) $\cdot B_{6}(\mathrm{O} 11)$ 
Energies: $\mathrm{G}_{\mathrm{gas}}=-591.106748$

$\mathrm{G}_{\mathrm{aq}}=-591.295590$

$\underline{\text { Coordinates }}$

\begin{tabular}{lrrr} 
& \multicolumn{1}{c}{$\mathrm{x}$} & $\mathrm{y}$ & \multicolumn{1}{c}{$\mathrm{z}$} \\
$\mathrm{C}$ & 0.056502 & 1.932283 & -0.028657 \\
$\mathrm{C}$ & -1.856011 & 0.631494 & -0.022975 \\
$\mathrm{C}$ & -1.071012 & -0.530402 & 0.173546 \\
$\mathrm{C}$ & 0.314784 & -0.406559 & 0.364028 \\
$\mathrm{C}$ & 0.916822 & 0.855377 & 0.189701 \\
$\mathrm{C}$ & -3.349423 & 0.528750 & -0.163240 \\
$\mathrm{C}$ & 1.093055 & -1.677892 & 0.726917 \\
$\mathrm{C}$ & 2.414613 & 1.060551 & 0.216416 \\
$\mathrm{O}$ & 0.970730 & -2.398796 & -0.447428 \\
$\mathrm{O}$ & -1.702578 & -1.731864 & 0.199017 \\
$\mathrm{O}$ & 3.058800 & 0.633025 & -0.985864 \\
$\mathrm{~N}$ & -1.279594 & 1.830192 & -0.115861 \\
$\mathrm{H}$ & 0.464999 & 2.934975 & -0.135473 \\
$\mathrm{H}$ & -3.619982 & -0.102119 & -1.017494 \\
$\mathrm{H}$ & -3.799973 & 0.067385 & 0.723011 \\
$\mathrm{H}$ & -3.765737 & 1.527357 & -0.303830 \\
$\mathrm{H}$ & -1.063527 & -2.422218 & -0.059571 \\
$\mathrm{H}$ & 2.134431 & -1.448141 & 0.982077 \\
$\mathrm{H}$ & 0.602031 & -2.176005 & 1.578547 \\
$\mathrm{H}$ & 2.774540 & -0.265002 & -1.206949 \\
$\mathrm{H}$ & 2.635739 & 2.127142 & 0.300863 \\
$\mathrm{H}$ & 2.863042 & 0.564749 & 1.089646
\end{tabular}

g) $\cdot \mathrm{B}_{6}(\mathrm{O} 12)$

Energies: $\mathrm{G}_{\mathrm{gas}}=-591.112319 \quad \mathrm{G}_{\mathrm{aq}}=-591.304911$

Coordinates

$\begin{array}{lrrr} & \mathrm{x} & \mathrm{y} & \mathrm{z} \\ \mathrm{C} & 1.844327 & -0.643892 & -0.034780 \\ \mathrm{~N} & 1.341324 & -1.872374 & 0.112686 \\ \mathrm{C} & 0.013234 & -2.015342 & 0.209678 \\ \mathrm{C} & -0.892546 & -0.955032 & 0.163204 \\ \mathrm{C} & -0.386260 & 0.351448 & 0.000000 \\ \mathrm{C} & 1.006992 & 0.494808 & -0.092180 \\ \mathrm{C} & -2.370605 & -1.257588 & 0.283831 \\ \mathrm{O} & -3.210601 & -0.693825 & -0.641509 \\ \mathrm{C} & -1.304120 & 1.546497 & -0.132321 \\ \mathrm{O} & -0.705302 & 2.688766 & 0.519127 \\ \mathrm{O} & 1.621861 & 1.698097 & -0.261623 \\ \mathrm{C} & 3.336096 & -0.488697 & -0.148491\end{array}$




$\begin{array}{rrrr}\mathrm{H} & -0.348800 & -3.035727 & 0.326557 \\ \mathrm{H} & 3.614398 & -0.037648 & -1.108059 \\ \mathrm{H} & 3.722857 & 0.176254 & 0.631973 \\ \mathrm{H} & 3.806927 & -1.469204 & -0.060136 \\ \mathrm{H} & 0.981510 & 2.401126 & -0.035690 \\ \mathrm{H} & -2.282367 & 1.345540 & 0.312108 \\ \mathrm{H} & -1.465598 & 1.767666 & -1.196878 \\ \mathrm{H} & -1.232174 & 3.474711 & 0.325868 \\ \mathrm{H} & -2.546796 & -2.348550 & 0.329729 \\ \mathrm{H} & -2.769606 & -0.905061 & 1.264015\end{array}$

h) $\mathrm{H}_{2} \mathrm{O}$

Energies: $\mathrm{G}_{\mathrm{gas}}=-76.429454 \quad \mathrm{G}_{\mathrm{aq}}=-76.469934$

$\underline{\text { Coordinates }}$

$\begin{array}{cccc} & \mathrm{x} & \mathrm{y} & \mathrm{z} \\ \mathrm{O} & 0.000000 & 0.000000 & 0.116380 \\ \mathrm{H} & 0.000000 & 0.769447 & -0.465518 \\ \mathrm{H} & 0.000000 & -0.769447 & -0.465518\end{array}$

\section{B 6 $+\cdot$ OOH -----> $\cdot \mathrm{B}_{6}+\mathrm{H}_{2} \mathrm{O}_{2}$ (2)}

\subsection{REACTANTS}

a) Pyridoxine

Energies: $\mathrm{G}_{\mathrm{gas}}=-591.770767 \quad \mathrm{G}_{\mathrm{aq}}=-591.967495$

Coordinates

\begin{tabular}{lrrr} 
& \multicolumn{1}{c}{$\mathrm{x}$} & $\mathrm{y}$ & \multicolumn{1}{c}{$\mathrm{z}$} \\
$\mathrm{C}$ & 0.010624 & 2.001714 & 0.073482 \\
$\mathrm{C}$ & -1.859630 & 0.662745 & -0.067344 \\
$\mathrm{C}$ & -1.060173 & -0.492804 & 0.093001 \\
$\mathrm{C}$ & 0.325908 & -0.373582 & 0.268084 \\
$\mathrm{C}$ & 0.880258 & 0.923474 & 0.247645 \\
$\mathrm{C}$ & -3.349532 & 0.537076 & -0.231889 \\
$\mathrm{C}$ & 1.159865 & -1.613381 & 0.525282 \\
$\mathrm{C}$ & 2.365388 & 1.175548 & 0.387882 \\
$\mathrm{O}$ & 0.780951 & -2.721212 & -0.321003 \\
$\mathrm{O}$ & -1.697245 & -1.697936 & 0.088458 \\
$\mathrm{O}$ & 3.133284 & 0.631117 & -0.697116 \\
$\mathrm{~N}$ & -1.316995 & 1.883493 & -0.072135 \\
$\mathrm{H}$ & 0.402989 & 3.017553 & 0.053538 \\
$\mathrm{H}$ & -3.599145 & -0.088961 & -1.096098
\end{tabular}




$\begin{array}{rrrr}\mathrm{H} & -3.804398 & 0.056403 & 0.642059 \\ \mathrm{H} & -3.781910 & 1.530398 & -0.363276 \\ \mathrm{H} & -1.030495 & -2.398743 & -0.054541 \\ \mathrm{H} & 2.224816 & -1.405823 & 0.409043 \\ \mathrm{H} & 0.986237 & -1.978769 & 1.544025 \\ \mathrm{H} & 1.091175 & -2.544588 & -1.219873 \\ \mathrm{H} & 2.543280 & 2.254822 & 0.482327 \\ \mathrm{H} & 2.775640 & 0.702345 & 1.285015 \\ \mathrm{H} & 2.838618 & 1.050425 & -1.516841\end{array}$

b) $\cdot \mathrm{OOH}$ Radical

Energies: $\mathrm{G}_{\mathrm{gas}}=-150.921387 \quad \mathrm{G}_{\mathrm{aq}}=-150.988356$

$\underline{\text { Coordinates }}$

$\begin{array}{cccc} & \mathrm{x} & \mathrm{y} & \mathrm{z} \\ \mathrm{O} & 0.055545 & 0.721264 & 0.000000 \\ \mathrm{O} & 0.055545 & -0.612053 & 0.000000 \\ \mathrm{H} & -0.888716 & -0.873690 & 0.000000\end{array}$

\subsection{PRODUCTS}
a) $\cdot \mathrm{B}_{6}(\mathrm{C} 6)$

Energies: $\mathrm{G}_{\mathrm{gas}}=-591.102531$

$$
\mathrm{G}_{\mathrm{aq}}=-591.298647
$$

$\underline{\text { Coordinates }}$

$\begin{array}{lrrr} & \mathrm{x} & \mathrm{y} & \mathrm{z} \\ \mathrm{C} & 1.190982 & 0.130104 & 0.124516 \\ \mathrm{C} & 1.460633 & -1.204672 & -0.229242 \\ \mathrm{~N} & 0.412095 & -2.028123 & -0.433032 \\ \mathrm{C} & -0.792898 & -1.607334 & -0.311912 \\ \mathrm{C} & -1.197534 & -0.315635 & 0.024488 \\ \mathrm{C} & -0.140305 & 0.584545 & 0.266607 \\ \mathrm{C} & 2.860750 & -1.723610 & -0.373572 \\ \mathrm{C} & -2.670705 & 0.016854 & 0.164463 \\ \mathrm{O} & -3.511670 & -0.929812 & -0.470741 \\ \mathrm{C} & -0.386016 & 2.008297 & 0.726723 \\ \mathrm{O} & 0.473440 & 2.964132 & 0.073369 \\ \mathrm{O} & 2.265071 & 0.943608 & 0.340033 \\ \mathrm{H} & 3.428390 & -1.126325 & -1.095562 \\ \mathrm{H} & 3.403189 & -1.665304 & 0.577248 \\ \mathrm{H} & 2.831972 & -2.763218 & -0.704721 \\ \mathrm{H} & 1.966868 & 1.873281 & 0.303960 \\ \mathrm{H} & -1.433120 & 2.297060 & 0.597857\end{array}$




$\begin{array}{rrrr}\mathrm{H} & -0.146012 & 2.107982 & 1.791213 \\ \mathrm{H} & 0.218150 & 3.035478 & -0.857056 \\ \mathrm{H} & -2.930342 & 0.098931 & 1.233431 \\ \mathrm{H} & -2.898693 & 0.985093 & -0.296148 \\ \mathrm{H} & -3.089246 & -1.800840 & -0.412715\end{array}$

b) $\cdot \mathrm{B}_{6}(\mathrm{C} 7)$

Energies: $\mathrm{G}_{\mathrm{gas}}=-591.131656 \quad \mathrm{G}_{\mathrm{aq}}=-591.329448$

Coordinates

\begin{tabular}{lrrr} 
& \multicolumn{1}{c}{$\mathrm{x}$} & $\mathrm{y}$ & \multicolumn{1}{c}{$\mathrm{z}$} \\
$\mathrm{N}$ & 1.343325 & -1.925135 & -0.041256 \\
$\mathrm{C}$ & 0.031623 & -2.011103 & 0.118317 \\
$\mathrm{C}$ & -0.837493 & -0.912741 & 0.272773 \\
$\mathrm{C}$ & -0.274591 & 0.383231 & 0.255621 \\
$\mathrm{C}$ & 1.106050 & 0.494615 & 0.068375 \\
$\mathrm{C}$ & 1.919757 & -0.683891 & -0.076370 \\
$\mathrm{C}$ & 3.306308 & -0.595612 & -0.253921 \\
$\mathrm{O}$ & 1.740913 & 1.692803 & 0.030339 \\
$\mathrm{C}$ & -1.105301 & 1.630285 & 0.491659 \\
$\mathrm{O}$ & -0.714622 & 2.728153 & -0.363215 \\
$\mathrm{C}$ & -2.320227 & -1.153158 & 0.431531 \\
$\mathrm{O}$ & -3.096488 & -0.631511 & -0.660610 \\
$\mathrm{H}$ & -0.382498 & -3.019676 & 0.132198 \\
$\mathrm{H}$ & 3.805936 & 0.363333 & -0.295972 \\
$\mathrm{H}$ & 3.880259 & -1.509341 & -0.350369 \\
$\mathrm{H}$ & 1.071101 & 2.393316 & -0.110988 \\
$\mathrm{H}$ & -2.169560 & 1.424875 & 0.366187 \\
$\mathrm{H}$ & -0.941352 & 2.006653 & 1.507925 \\
$\mathrm{H}$ & -1.024143 & 2.547393 & -1.261593 \\
$\mathrm{H}$ & -2.504160 & -2.228466 & 0.554692 \\
$\mathrm{H}$ & -2.721666 & -0.654415 & 1.319163 \\
$\mathrm{H}$ & -2.812360 & -1.073051 & -1.472475
\end{tabular}

c) $\cdot \mathrm{B}_{6}(\mathrm{C} 8)$

Energies: $\mathrm{G}_{\mathrm{gas}}=-591.144028 \quad \mathrm{G}_{\mathrm{aq}}=-591.345021$

Coordinates

$\begin{array}{crrr} & \mathrm{x} & \mathrm{y} & \mathrm{z} \\ \mathrm{C} & 1.070991 & 0.491498 & 0.000000 \\ \mathrm{C} & 1.836954 & -0.688778 & -0.037959 \\ \mathrm{~N} & 1.275621 & -1.901801 & 0.042370 \\ \mathrm{C} & -0.065036 & -1.977337 & 0.162739\end{array}$




$\begin{array}{rrrr}\mathrm{C} & -0.920749 & -0.889490 & 0.209756 \\ \mathrm{C} & -0.352842 & 0.430143 & 0.111531 \\ \mathrm{C} & 3.334196 & -0.607794 & -0.172870 \\ \mathrm{C} & -2.400337 & -1.107174 & 0.401947 \\ \mathrm{O} & -3.215239 & -0.593569 & -0.665708 \\ \mathrm{C} & -1.189497 & 1.559986 & 0.123853 \\ \mathrm{O} & -0.640439 & 2.823499 & 0.137653 \\ \mathrm{O} & 1.759630 & 1.662090 & -0.109712 \\ \mathrm{H} & -0.474151 & -2.984014 & 0.236868 \\ \mathrm{H} & 3.621669 & -0.096016 & -1.098592 \\ \mathrm{H} & 3.775906 & -0.035509 & 0.650911 \\ \mathrm{H} & 3.746468 & -1.618191 & -0.174740 \\ \mathrm{H} & 1.145878 & 2.413267 & -0.065477 \\ \mathrm{H} & -2.268367 & 1.489913 & 0.094630 \\ \mathrm{H} & -1.321843 & 3.496248 & 0.019237 \\ \mathrm{H} & -2.591734 & -2.178445 & 0.547513 \\ \mathrm{H} & -2.761708 & -0.585711 & 1.295514 \\ \mathrm{H} & -2.915160 & -0.991414 & -1.494219\end{array}$
d) $\cdot B_{6}(C 9)$

Energies: $\mathrm{G}_{\mathrm{gas}}=-591.139957 \quad \mathrm{G}_{\mathrm{aq}}=-591.344235$

\section{$\underline{\text { Coordinates }}$}

\begin{tabular}{lrrr} 
& \multicolumn{1}{c}{$\mathrm{x}$} & $\mathrm{y}$ & \multicolumn{1}{c}{$\mathrm{z}$} \\
$\mathrm{C}$ & -1.171110 & 0.133971 & 0.074321 \\
$\mathrm{C}$ & -1.388579 & -1.264379 & -0.011621 \\
$\mathrm{~N}$ & -0.360966 & -2.123724 & -0.087273 \\
$\mathrm{C}$ & 0.883554 & -1.656782 & -0.078434 \\
$\mathrm{C}$ & 1.216000 & -0.273316 & 0.000000 \\
$\mathrm{C}$ & 0.124245 & 0.655150 & 0.079385 \\
$\mathrm{C}$ & -2.785932 & -1.817804 & -0.015758 \\
$\mathrm{C}$ & 2.568934 & 0.120642 & 0.053124 \\
$\mathrm{O}$ & 3.541630 & -0.839051 & -0.032143 \\
$\mathrm{C}$ & 0.386217 & 2.134930 & 0.233865 \\
$\mathrm{O}$ & -0.651257 & 2.899140 & -0.416667 \\
$\mathrm{O}$ & -2.292031 & 0.908068 & 0.166969 \\
$\mathrm{H}$ & 1.680588 & -2.390501 & -0.140538 \\
$\mathrm{H}$ & -3.320692 & -1.561065 & 0.906882 \\
$\mathrm{H}$ & -3.375852 & -1.402006 & -0.841157 \\
$\mathrm{H}$ & -2.739160 & -2.903974 & -0.113362 \\
$\mathrm{H}$ & -2.040739 & 1.833519 & -0.013772 \\
$\mathrm{H}$ & 1.347679 & 2.402077 & -0.217134 \\
$\mathrm{H}$ & 0.423388 & 2.403278 & 1.301748 \\
$\mathrm{H}$ & -0.555348 & 3.830695 & -0.181192 \\
$\mathrm{H}$ & 2.905820 & 1.144924 & 0.153764 \\
$\mathrm{H}$ & 4.414370 & -0.430599 & 0.000000
\end{tabular}


e) $\cdot \mathrm{B}_{6}(\mathrm{O} 10)$

Energies: $\mathrm{G}_{\mathrm{gas}}=-591.140797 \quad \mathrm{G}_{\mathrm{aq}}=-591.334827$

$\underline{\text { Coordinates }}$

\begin{tabular}{lrrr} 
& \multicolumn{1}{c}{$\mathrm{x}$} & $\mathrm{y}$ & $\mathrm{z}$ \\
$\mathrm{C}$ & -0.166125 & -1.880899 & 0.000000 \\
$\mathrm{C}$ & 1.829320 & -0.751360 & -0.063629 \\
$\mathrm{C}$ & 1.137534 & 0.520425 & 0.184791 \\
$\mathrm{C}$ & -0.310929 & 0.493342 & 0.361642 \\
$\mathrm{C}$ & -0.956584 & -0.727450 & 0.259446 \\
$\mathrm{C}$ & 3.314181 & -0.749920 & -0.235920 \\
$\mathrm{C}$ & -0.971663 & 1.829527 & 0.632999 \\
$\mathrm{C}$ & -2.459578 & -0.914251 & 0.343037 \\
$\mathrm{O}$ & -0.743264 & 2.759247 & -0.420234 \\
$\mathrm{O}$ & 1.781418 & 1.601407 & 0.229966 \\
$\mathrm{O}$ & -3.057205 & -1.016712 & -0.950724 \\
$\mathrm{~N}$ & 1.168748 & -1.901499 & -0.148166 \\
$\mathrm{H}$ & -0.662487 & -2.845637 & -0.089088 \\
$\mathrm{H}$ & 3.602406 & -0.122546 & -1.087549 \\
$\mathrm{H}$ & 3.805552 & -0.312779 & 0.640844 \\
$\mathrm{H}$ & 3.667492 & -1.769941 & -0.392109 \\
$\mathrm{H}$ & 0.212836 & 2.925664 & -0.436931 \\
$\mathrm{H}$ & -2.054152 & 1.730650 & 0.721516 \\
$\mathrm{H}$ & -0.594988 & 2.222999 & 1.588839 \\
$\mathrm{H}$ & -2.687188 & -1.862904 & 0.836970 \\
$\mathrm{H}$ & -2.930773 & -0.120083 & 0.932781 \\
$\mathrm{H}$ & -2.884464 & -0.202953 & -1.444375
\end{tabular}

f) $\cdot B_{6}(\mathrm{O} 11)$

Energies: $\mathrm{G}_{\mathrm{gas}}=-591.106748 \quad \mathrm{G}_{\mathrm{aq}}=-591.295590$

$\underline{\text { Coordinates }}$

$\begin{array}{crrr} & \mathrm{x} & \mathrm{y} & \mathrm{z} \\ \mathrm{C} & 0.056502 & 1.932283 & -0.028657 \\ \mathrm{C} & -1.856011 & 0.631494 & -0.022975 \\ \mathrm{C} & -1.071012 & -0.530402 & 0.173546 \\ \mathrm{C} & 0.314784 & -0.406559 & 0.364028 \\ \mathrm{C} & 0.916822 & 0.855377 & 0.189701 \\ \mathrm{C} & -3.349423 & 0.528750 & -0.163240 \\ \mathrm{C} & 1.093055 & -1.677892 & 0.726917 \\ \mathrm{C} & 2.414613 & 1.060551 & 0.216416 \\ \mathrm{O} & 0.970730 & -2.398796 & -0.447428\end{array}$




$\begin{array}{rrrr}\mathrm{O} & -1.702578 & -1.731864 & 0.199017 \\ \mathrm{O} & 3.058800 & 0.633025 & -0.985864 \\ \mathrm{~N} & -1.279594 & 1.830192 & -0.115861 \\ \mathrm{H} & 0.464999 & 2.934975 & -0.135473 \\ \mathrm{H} & -3.619982 & -0.102119 & -1.017494 \\ \mathrm{H} & -3.799973 & 0.067385 & 0.723011 \\ \mathrm{H} & -3.765737 & 1.527357 & -0.303830 \\ \mathrm{H} & -1.063527 & -2.422218 & -0.059571 \\ \mathrm{H} & 2.134431 & -1.448141 & 0.982077 \\ \mathrm{H} & 0.602031 & -2.176005 & 1.578547 \\ \mathrm{H} & 2.774540 & -0.265002 & -1.206949 \\ \mathrm{H} & 2.635739 & 2.127142 & 0.300863 \\ \mathrm{H} & 2.863042 & 0.564749 & 1.089646\end{array}$

$$
\text { g) } \cdot \mathrm{B}_{6}(\mathrm{O} 12)
$$

Energies: $\mathrm{G}_{\mathrm{gas}}=-591.112319 \quad \mathrm{G}_{\mathrm{aq}}=-591.304911$

$\underline{\text { Coordinates }}$

\begin{tabular}{lrrr} 
& \multicolumn{1}{c}{$\mathrm{x}$} & $\mathrm{y}$ & \multicolumn{1}{c}{$\mathrm{z}$} \\
$\mathrm{C}$ & 1.844327 & -0.643892 & -0.034780 \\
$\mathrm{~N}$ & 1.341324 & -1.872374 & 0.112686 \\
$\mathrm{C}$ & 0.013234 & -2.015342 & 0.209678 \\
$\mathrm{C}$ & -0.892546 & -0.955032 & 0.163204 \\
$\mathrm{C}$ & -0.386260 & 0.351448 & 0.000000 \\
$\mathrm{C}$ & 1.006992 & 0.494808 & -0.092180 \\
$\mathrm{C}$ & -2.370605 & -1.257588 & 0.283831 \\
$\mathrm{O}$ & -3.210601 & -0.693825 & -0.641509 \\
$\mathrm{C}$ & -1.304120 & 1.546497 & -0.132321 \\
$\mathrm{O}$ & -0.705302 & 2.688766 & 0.519127 \\
$\mathrm{O}$ & 1.621861 & 1.698097 & -0.261623 \\
$\mathrm{C}$ & 3.336096 & -0.488697 & -0.148491 \\
$\mathrm{H}$ & -0.348800 & -3.035727 & 0.326557 \\
$\mathrm{H}$ & 3.614398 & -0.037648 & -1.108059 \\
$\mathrm{H}$ & 3.722857 & 0.176254 & 0.631973 \\
$\mathrm{H}$ & 3.806927 & -1.469204 & -0.060136 \\
$\mathrm{H}$ & 0.981510 & 2.401126 & -0.035690 \\
$\mathrm{H}$ & -2.282367 & 1.345540 & 0.312108 \\
$\mathrm{H}$ & -1.465598 & 1.767666 & -1.196878 \\
$\mathrm{H}$ & -1.232174 & 3.474711 & 0.325868 \\
$\mathrm{H}$ & -2.546796 & -2.348550 & 0.329729 \\
$\mathrm{H}$ & -2.769606 & -0.905061 & 1.264015
\end{tabular}

h) $\mathrm{H}_{2} \mathrm{O}_{2}$

Energies: $\mathrm{G}_{\mathrm{gas}}=-151.550773 \quad \mathrm{G}_{\mathrm{aq}}=-151.622535$ 


\section{$\underline{\text { Coordinates }}$}

$\begin{array}{cccc} & \mathrm{x} & \mathrm{y} & \mathrm{z} \\ \mathrm{O} & 0.000000 & 0.733410 & 0.000000 \\ \mathrm{O} & 0.000000 & -0.733410 & 0.000000 \\ \mathrm{H} & 0.957943 & 0.883655 & 0.000000 \\ \mathrm{H} & -0.957943 & -0.883655 & 0.000000\end{array}$

\section{3. $\mathrm{B}_{6}+\cdot \mathrm{O}_{2}^{-}-\cdot--->\cdot \mathrm{B}_{6}+\mathrm{OOH}^{-}$(3)}

\subsection{REACTANTS}

a) Pyridoxine

Energies: $\mathrm{G}_{\mathrm{gas}}=-591.770767 \quad \mathrm{G}_{\mathrm{aq}}=-591.967495$

$\underline{\text { Coordinates }}$

\begin{tabular}{lrrr} 
& \multicolumn{1}{c}{$\mathrm{x}$} & $\mathrm{y}$ & \multicolumn{1}{c}{$\mathrm{z}$} \\
$\mathrm{C}$ & 0.010624 & 2.001714 & 0.073482 \\
$\mathrm{C}$ & -1.859630 & 0.662745 & -0.067344 \\
$\mathrm{C}$ & -1.060173 & -0.492804 & 0.093001 \\
$\mathrm{C}$ & 0.325908 & -0.373582 & 0.268084 \\
$\mathrm{C}$ & 0.880258 & 0.923474 & 0.247645 \\
$\mathrm{C}$ & -3.349532 & 0.537076 & -0.231889 \\
$\mathrm{C}$ & 1.159865 & -1.613381 & 0.525282 \\
$\mathrm{C}$ & 2.365388 & 1.175548 & 0.387882 \\
$\mathrm{O}$ & 0.780951 & -2.721212 & -0.321003 \\
$\mathrm{O}$ & -1.697245 & -1.697936 & 0.088458 \\
$\mathrm{O}$ & 3.133284 & 0.631117 & -0.697116 \\
$\mathrm{~N}$ & -1.316995 & 1.883493 & -0.072135 \\
$\mathrm{H}$ & 0.402989 & 3.017553 & 0.053538 \\
$\mathrm{H}$ & -3.599145 & -0.088961 & -1.096098 \\
$\mathrm{H}$ & -3.804398 & 0.056403 & 0.642059 \\
$\mathrm{H}$ & -3.781910 & 1.530398 & -0.363276 \\
$\mathrm{H}$ & -1.030495 & -2.398743 & -0.054541 \\
$\mathrm{H}$ & 2.224816 & -1.405823 & 0.409043 \\
$\mathrm{H}$ & 0.986237 & -1.978769 & 1.544025 \\
$\mathrm{H}$ & 1.091175 & -2.544588 & -1.219873 \\
$\mathrm{H}$ & 2.543280 & 2.254822 & 0.482327 \\
$\mathrm{H}$ & 2.775640 & 0.702345 & 1.285015 \\
$\mathrm{H}$ & 2.838618 & 1.050425 & -1.516841
\end{tabular}

b) $\cdot \mathrm{O}_{2}^{-}$Radical

Energies: $\mathrm{G}_{\mathrm{gas}}=-150.364299 \quad \mathrm{G}_{\mathrm{aq}}=-150.535287$ 
$\underline{\text { Coordinates }}$

$\begin{array}{cccc} & \mathrm{x} & \mathrm{y} & \mathrm{z} \\ \mathrm{O} & 0.000000 & 0.000000 & 0.675349 \\ \mathrm{O} & 0.000000 & 0.000000 & -0.675349\end{array}$

\subsection{PRODUCTS}
a) $\cdot B_{6}(C 6)$

Energies: $\mathrm{G}_{\mathrm{gas}}=-591.102531$

$$
\mathrm{G}_{\mathrm{aq}}=-591.298647
$$

$\underline{\text { Coordinates }}$

$\begin{array}{lrrr} & \mathrm{X} & \mathrm{y} & \mathrm{z} \\ \mathrm{C} & 1.190982 & 0.130104 & 0.124516 \\ \mathrm{C} & 1.460633 & -1.204672 & -0.229242 \\ \mathrm{~N} & 0.412095 & -2.028123 & -0.433032 \\ \mathrm{C} & -0.792898 & -1.607334 & -0.311912 \\ \mathrm{C} & -1.197534 & -0.315635 & 0.024488 \\ \mathrm{C} & -0.140305 & 0.584545 & 0.266607 \\ \mathrm{C} & 2.860750 & -1.723610 & -0.373572 \\ \mathrm{C} & -2.670705 & 0.016854 & 0.164463 \\ \mathrm{O} & -3.511670 & -0.929812 & -0.470741 \\ \mathrm{C} & -0.386016 & 2.008297 & 0.726723 \\ \mathrm{O} & 0.473440 & 2.964132 & 0.073369 \\ \mathrm{O} & 2.265071 & 0.943608 & 0.340033 \\ \mathrm{H} & 3.428390 & -1.126325 & -1.095562 \\ \mathrm{H} & 3.403189 & -1.665304 & 0.577248 \\ \mathrm{H} & 2.831972 & -2.763218 & -0.704721 \\ \mathrm{H} & 1.966868 & 1.873281 & 0.303960 \\ \mathrm{H} & -1.433120 & 2.297060 & 0.597857 \\ \mathrm{H} & -0.146012 & 2.107982 & 1.791213 \\ \mathrm{H} & 0.218150 & 3.035478 & -0.857056 \\ \mathrm{H} & -2.930342 & 0.098931 & 1.233431 \\ \mathrm{H} & -2.898693 & 0.985093 & -0.296148 \\ \mathrm{H} & -3.089246 & -1.800840 & -0.412715\end{array}$

b) $\cdot B_{6}(C 7)$

Energies: $\mathrm{G}_{\mathrm{gas}}=-591.131656 \quad \mathrm{G}_{\mathrm{aq}}=-591.329448$

$\underline{\text { Coordinates }}$

$\begin{array}{cccc} & \mathrm{X} & \mathrm{y} & \mathrm{z} \\ \mathrm{N} & 1.343325 & -1.925135 & -0.041256\end{array}$ 


$\begin{array}{lrrr}\mathrm{C} & 0.031623 & -2.011103 & 0.118317 \\ \mathrm{C} & -0.837493 & -0.912741 & 0.272773 \\ \mathrm{C} & -0.274591 & 0.383231 & 0.255621 \\ \mathrm{C} & 1.106050 & 0.494615 & 0.068375 \\ \mathrm{C} & 1.919757 & -0.683891 & -0.076370 \\ \mathrm{C} & 3.306308 & -0.595612 & -0.253921 \\ \mathrm{O} & 1.740913 & 1.692803 & 0.030339 \\ \mathrm{C} & -1.105301 & 1.630285 & 0.491659 \\ \mathrm{O} & -0.714622 & 2.728153 & -0.363215 \\ \mathrm{C} & -2.320227 & -1.153158 & 0.431531 \\ \mathrm{O} & -3.096488 & -0.631511 & -0.660610 \\ \mathrm{H} & -0.382498 & -3.019676 & 0.132198 \\ \mathrm{H} & 3.805936 & 0.363333 & -0.295972 \\ \mathrm{H} & 3.880259 & -1.509341 & -0.350369 \\ \mathrm{H} & 1.071101 & 2.393316 & -0.110988 \\ \mathrm{H} & -2.169560 & 1.424875 & 0.366187 \\ \mathrm{H} & -0.941352 & 2.006653 & 1.507925 \\ \mathrm{H} & -1.024143 & 2.547393 & -1.261593 \\ \mathrm{H} & -2.504160 & -2.228466 & 0.554692 \\ \mathrm{H} & -2.721666 & -0.654415 & 1.319163 \\ \mathrm{H} & -2.812360 & -1.073051 & -1.472475\end{array}$

c) $\cdot \mathrm{B}_{6}(\mathrm{C} 8)$

Energies: $\mathrm{G}_{\mathrm{gas}}=-591.144028 \quad \mathrm{G}_{\mathrm{aq}}=-591.345021$

$\underline{\text { Coordinates }}$

\begin{tabular}{lrrr} 
& \multicolumn{1}{c}{$\mathrm{x}$} & $\mathrm{y}$ & \multicolumn{1}{c}{$\mathrm{z}$} \\
$\mathrm{C}$ & 1.070991 & 0.491498 & 0.000000 \\
$\mathrm{C}$ & 1.836954 & -0.688778 & -0.037959 \\
$\mathrm{~N}$ & 1.275621 & -1.901801 & 0.042370 \\
$\mathrm{C}$ & -0.065036 & -1.977337 & 0.162739 \\
$\mathrm{C}$ & -0.920749 & -0.889490 & 0.209756 \\
$\mathrm{C}$ & -0.352842 & 0.430143 & 0.111531 \\
$\mathrm{C}$ & 3.334196 & -0.607794 & -0.172870 \\
$\mathrm{C}$ & -2.400337 & -1.107174 & 0.401947 \\
$\mathrm{O}$ & -3.215239 & -0.593569 & -0.665708 \\
$\mathrm{C}$ & -1.189497 & 1.559986 & 0.123853 \\
$\mathrm{O}$ & -0.640439 & 2.823499 & 0.137653 \\
$\mathrm{O}$ & 1.759630 & 1.662090 & -0.109712 \\
$\mathrm{H}$ & -0.474151 & -2.984014 & 0.236868 \\
$\mathrm{H}$ & 3.621669 & -0.096016 & -1.098592 \\
$\mathrm{H}$ & 3.775906 & -0.035509 & 0.650911 \\
$\mathrm{H}$ & 3.746468 & -1.618191 & -0.174740 \\
$\mathrm{H}$ & 1.145878 & 2.413267 & -0.065477 \\
$\mathrm{H}$ & -2.268367 & 1.489913 & 0.094630 \\
$\mathrm{H}$ & -1.321843 & 3.496248 & 0.019237
\end{tabular}


$\begin{array}{lrrr}\mathrm{H} & -2.591734 & -2.178445 & 0.547513 \\ \mathrm{H} & -2.761708 & -0.585711 & 1.295514 \\ \mathrm{H} & -2.915160 & -0.991414 & -1.494219\end{array}$

d) $\cdot B_{6}(C 9)$

Energies: $\mathrm{G}_{\mathrm{gas}}=-591.139957 \quad \mathrm{G}_{\mathrm{aq}}=-591.344235$

$\underline{\text { Coordinates }}$

\begin{tabular}{lrrr} 
& \multicolumn{1}{c}{$\mathrm{x}$} & $\mathrm{y}$ & \multicolumn{1}{c}{$\mathrm{z}$} \\
$\mathrm{C}$ & -1.171110 & 0.133971 & 0.074321 \\
$\mathrm{C}$ & -1.388579 & -1.264379 & -0.011621 \\
$\mathrm{~N}$ & -0.360966 & -2.123724 & -0.087273 \\
$\mathrm{C}$ & 0.883554 & -1.656782 & -0.078434 \\
$\mathrm{C}$ & 1.216000 & -0.273316 & 0.000000 \\
$\mathrm{C}$ & 0.124245 & 0.655150 & 0.079385 \\
$\mathrm{C}$ & -2.785932 & -1.817804 & -0.015758 \\
$\mathrm{C}$ & 2.568934 & 0.120642 & 0.053124 \\
$\mathrm{O}$ & 3.541630 & -0.839051 & -0.032143 \\
$\mathrm{C}$ & 0.386217 & 2.134930 & 0.233865 \\
$\mathrm{O}$ & -0.651257 & 2.899140 & -0.416667 \\
$\mathrm{O}$ & -2.292031 & 0.908068 & 0.166969 \\
$\mathrm{H}$ & 1.680588 & -2.390501 & -0.140538 \\
$\mathrm{H}$ & -3.320692 & -1.561065 & 0.906882 \\
$\mathrm{H}$ & -3.375852 & -1.402006 & -0.841157 \\
$\mathrm{H}$ & -2.739160 & -2.903974 & -0.113362 \\
$\mathrm{H}$ & -2.040739 & 1.833519 & -0.013772 \\
$\mathrm{H}$ & 1.347679 & 2.402077 & -0.217134 \\
$\mathrm{H}$ & 0.423388 & 2.403278 & 1.301748 \\
$\mathrm{H}$ & -0.555348 & 3.830695 & -0.181192 \\
$\mathrm{H}$ & 2.905820 & 1.144924 & 0.153764 \\
$\mathrm{H}$ & 4.414370 & -0.430599 & 0.000000
\end{tabular}

e) $\cdot \mathrm{B}_{6}(\mathrm{O} 10)$

Energies: $\mathrm{G}_{\mathrm{gas}}=-591.140797 \quad \mathrm{G}_{\mathrm{aq}}=-591.334827$

$\underline{\text { Coordinates }}$

$\begin{array}{crrr} & & & \\ & \mathrm{x} & \mathrm{y} & \mathrm{z} \\ \mathrm{C} & -0.166125 & -1.880899 & 0.000000 \\ \mathrm{C} & 1.829320 & -0.751360 & -0.063629 \\ \mathrm{C} & 1.137534 & 0.520425 & 0.184791 \\ \mathrm{C} & -0.310929 & 0.493342 & 0.361642 \\ \mathrm{C} & -0.956584 & -0.727450 & 0.259446 \\ \mathrm{C} & 3.314181 & -0.749920 & -0.235920\end{array}$




$\begin{array}{rrrr}\mathrm{C} & -0.971663 & 1.829527 & 0.632999 \\ \mathrm{C} & -2.459578 & -0.914251 & 0.343037 \\ \mathrm{O} & -0.743264 & 2.759247 & -0.420234 \\ \mathrm{O} & 1.781418 & 1.601407 & 0.229966 \\ \mathrm{O} & -3.057205 & -1.016712 & -0.950724 \\ \mathrm{~N} & 1.168748 & -1.901499 & -0.148166 \\ \mathrm{H} & -0.662487 & -2.845637 & -0.089088 \\ \mathrm{H} & 3.602406 & -0.122546 & -1.087549 \\ \mathrm{H} & 3.805552 & -0.312779 & 0.640844 \\ \mathrm{H} & 3.667492 & -1.769941 & -0.392109 \\ \mathrm{H} & 0.212836 & 2.925664 & -0.436931 \\ \mathrm{H} & -2.054152 & 1.730650 & 0.721516 \\ \mathrm{H} & -0.594988 & 2.222999 & 1.588839 \\ \mathrm{H} & -2.687188 & -1.862904 & 0.836970 \\ \mathrm{H} & -2.930773 & -0.120083 & 0.932781 \\ \mathrm{H} & -2.884464 & -0.202953 & -1.444375\end{array}$

f) $\cdot \mathrm{B}_{6}(\mathrm{O} 11)$

Energies: $\mathrm{G}_{\mathrm{gas}}=-591.106748 \quad \mathrm{G}_{\mathrm{aq}}=-591.295590$

$\underline{\text { Coordinates }}$

\begin{tabular}{lrrr} 
& \multicolumn{1}{c}{$\mathrm{x}$} & $\mathrm{y}$ & \multicolumn{1}{c}{$\mathrm{z}$} \\
$\mathrm{C}$ & 0.056502 & 1.932283 & -0.028657 \\
$\mathrm{C}$ & -1.856011 & 0.631494 & -0.022975 \\
$\mathrm{C}$ & -1.071012 & -0.530402 & 0.173546 \\
$\mathrm{C}$ & 0.314784 & -0.406559 & 0.364028 \\
$\mathrm{C}$ & 0.916822 & 0.855377 & 0.189701 \\
$\mathrm{C}$ & -3.349423 & 0.528750 & -0.163240 \\
$\mathrm{C}$ & 1.093055 & -1.677892 & 0.726917 \\
$\mathrm{C}$ & 2.414613 & 1.060551 & 0.216416 \\
$\mathrm{O}$ & 0.970730 & -2.398796 & -0.447428 \\
$\mathrm{O}$ & -1.702578 & -1.731864 & 0.199017 \\
$\mathrm{O}$ & 3.058800 & 0.633025 & -0.985864 \\
$\mathrm{~N}$ & -1.279594 & 1.830192 & -0.115861 \\
$\mathrm{H}$ & 0.464999 & 2.934975 & -0.135473 \\
$\mathrm{H}$ & -3.619982 & -0.102119 & -1.017494 \\
$\mathrm{H}$ & -3.799973 & 0.067385 & 0.723011 \\
$\mathrm{H}$ & -3.765737 & 1.527357 & -0.303830 \\
$\mathrm{H}$ & -1.063527 & -2.422218 & -0.059571 \\
$\mathrm{H}$ & 2.134431 & -1.448141 & 0.982077 \\
$\mathrm{H}$ & 0.602031 & -2.176005 & 1.578547 \\
$\mathrm{H}$ & 2.774540 & -0.265002 & -1.206949 \\
$\mathrm{H}$ & 2.635739 & 2.127142 & 0.300863 \\
$\mathrm{H}$ & 2.863042 & 0.564749 & 1.089646
\end{tabular}




$$
\text { g) } \cdot \mathrm{B}_{6}(\mathrm{O} 12)
$$

Energies: $\mathrm{G}_{\mathrm{gas}}=-591.112319 \quad \mathrm{G}_{\mathrm{aq}}=-591.304911$

$\underline{\text { Coordinates }}$

\begin{tabular}{lrrr} 
& \multicolumn{1}{c}{$\mathrm{x}$} & $\mathrm{y}$ & \multicolumn{1}{c}{$\mathrm{z}$} \\
$\mathrm{C}$ & 1.844327 & -0.643892 & -0.034780 \\
$\mathrm{~N}$ & 1.341324 & -1.872374 & 0.112686 \\
$\mathrm{C}$ & 0.013234 & -2.015342 & 0.209678 \\
$\mathrm{C}$ & -0.892546 & -0.955032 & 0.163204 \\
$\mathrm{C}$ & -0.386260 & 0.351448 & 0.000000 \\
$\mathrm{C}$ & 1.006992 & 0.494808 & -0.092180 \\
$\mathrm{C}$ & -2.370605 & -1.257588 & 0.283831 \\
$\mathrm{O}$ & -3.210601 & -0.693825 & -0.641509 \\
$\mathrm{C}$ & -1.304120 & 1.546497 & -0.132321 \\
$\mathrm{O}$ & -0.705302 & 2.688766 & 0.519127 \\
$\mathrm{O}$ & 1.621861 & 1.698097 & -0.261623 \\
$\mathrm{C}$ & 3.336096 & -0.488697 & -0.148491 \\
$\mathrm{H}$ & -0.348800 & -3.035727 & 0.326557 \\
$\mathrm{H}$ & 3.614398 & -0.037648 & -1.108059 \\
$\mathrm{H}$ & 3.722857 & 0.176254 & 0.631973 \\
$\mathrm{H}$ & 3.806927 & -1.469204 & -0.060136 \\
$\mathrm{H}$ & 0.981510 & 2.401126 & -0.035690 \\
$\mathrm{H}$ & -2.282367 & 1.345540 & 0.312108 \\
$\mathrm{H}$ & -1.465598 & 1.767666 & -1.196878 \\
$\mathrm{H}$ & -1.232174 & 3.474711 & 0.325868 \\
$\mathrm{H}$ & -2.546796 & -2.348550 & 0.329729 \\
$\mathrm{H}$ & -2.769606 & -0.905061 & 1.264015
\end{tabular}

h) $\mathrm{OOH}^{-}$

Energies: $\mathrm{G}_{\mathrm{gas}}=-150.958728 \quad \mathrm{G}_{\mathrm{aq}}=-151.142397$

$\underline{\text { Coordinates }}$

$\begin{array}{cccc} & \mathrm{x} & \mathrm{y} & \mathrm{z} \\ \mathrm{O} & -0.056081 & 0.814266 & 0.000000 \\ \mathrm{O} & -0.056081 & -0.707099 & 0.000000 \\ \mathrm{H} & 0.897293 & -0.857337 & 0.000000\end{array}$




\section{B $\mathrm{B}_{6}+\cdot \mathrm{OH}----->\mathrm{B}_{6}^{-}+\cdot \mathrm{OH}_{2}{ }^{+}(4)$}

\subsection{REACTANTS}

a) Pyridoxine

Energies: $\mathrm{G}_{\mathrm{gas}}=-591.770767 \quad \mathrm{G}_{\mathrm{aq}}=-591.967495$

$\underline{\text { Coordinates }}$

\begin{tabular}{lrrr} 
& \multicolumn{1}{c}{$\mathrm{x}$} & $\mathrm{y}$ & \multicolumn{1}{c}{$\mathrm{z}$} \\
$\mathrm{C}$ & 0.010624 & 2.001714 & 0.073482 \\
$\mathrm{C}$ & -1.859630 & 0.662745 & -0.067344 \\
$\mathrm{C}$ & -1.060173 & -0.492804 & 0.093001 \\
$\mathrm{C}$ & 0.325908 & -0.373582 & 0.268084 \\
$\mathrm{C}$ & 0.880258 & 0.923474 & 0.247645 \\
$\mathrm{C}$ & -3.349532 & 0.537076 & -0.231889 \\
$\mathrm{C}$ & 1.159865 & -1.613381 & 0.525282 \\
$\mathrm{C}$ & 2.365388 & 1.175548 & 0.387882 \\
$\mathrm{O}$ & 0.780951 & -2.721212 & -0.321003 \\
$\mathrm{O}$ & -1.697245 & -1.697936 & 0.088458 \\
$\mathrm{O}$ & 3.133284 & 0.631117 & -0.697116 \\
$\mathrm{~N}$ & -1.316995 & 1.883493 & -0.072135 \\
$\mathrm{H}$ & 0.402989 & 3.017553 & 0.053538 \\
$\mathrm{H}$ & -3.599145 & -0.088961 & -1.096098 \\
$\mathrm{H}$ & -3.804398 & 0.056403 & 0.642059 \\
$\mathrm{H}$ & -3.781910 & 1.530398 & -0.363276 \\
$\mathrm{H}$ & -1.030495 & -2.398743 & -0.054541 \\
$\mathrm{H}$ & 2.224816 & -1.405823 & 0.409043 \\
$\mathrm{H}$ & 0.986237 & -1.978769 & 1.544025 \\
$\mathrm{H}$ & 1.091175 & -2.544588 & -1.219873 \\
$\mathrm{H}$ & 2.543280 & 2.254822 & 0.482327 \\
$\mathrm{H}$ & 2.775640 & 0.702345 & 1.285015 \\
$\mathrm{H}$ & 2.838618 & 1.050425 & -1.516841
\end{tabular}

b) $\cdot \mathrm{OH}$ Radical

Energies: $\mathrm{G}_{\mathrm{gas}}=-75.746489 \quad \mathrm{G}_{\mathrm{aq}}=-75.784166$

$\underline{\text { Coordinates }}$
$\begin{array}{cccc} & \mathrm{x} & \mathrm{y} & \mathrm{z} \\ \mathrm{O} & 0.000000 & 0.000000 & 0.108834\end{array}$
$\begin{array}{llll}\mathrm{H} & 0.000000 & 0.000000 & -0.870670\end{array}$ 


\subsection{PRODUCTS}

a) $\mathrm{B}_{6}^{-}(\mathrm{C} 6)$

Energies: $\mathrm{G}_{\mathrm{gas}}=-591.158825 \quad \mathrm{G}_{\mathrm{aq}}=-591.429972$

$\underline{\text { Coordinates }}$

\begin{tabular}{lrrr} 
& \multicolumn{1}{c}{$\mathrm{x}$} & $\mathrm{y}$ & $\mathrm{z}$ \\
$\mathrm{C}$ & 1.195700 & 0.107914 & 0.149921 \\
$\mathrm{C}$ & 1.381240 & -1.219399 & -0.250671 \\
$\mathrm{~N}$ & 0.341434 & -2.039237 & -0.516066 \\
$\mathrm{C}$ & -0.952785 & -1.606013 & -0.404691 \\
$\mathrm{C}$ & -1.163834 & -0.269105 & 0.012859 \\
$\mathrm{C}$ & -0.109289 & 0.609312 & 0.299976 \\
$\mathrm{C}$ & 2.776841 & -1.781249 & -0.398778 \\
$\mathrm{C}$ & -2.647417 & 0.112903 & 0.123223 \\
$\mathrm{O}$ & -3.434817 & -0.978625 & -0.283853 \\
$\mathrm{C}$ & -0.312457 & 2.026325 & 0.773718 \\
$\mathrm{O}$ & 0.456691 & 2.996404 & 0.000000 \\
$\mathrm{O}$ & 2.315627 & 0.897259 & 0.403931 \\
$\mathrm{H}$ & 3.379838 & -1.193883 & -1.104079 \\
$\mathrm{H}$ & 3.322450 & -1.778119 & 0.554863 \\
$\mathrm{H}$ & 2.696471 & -2.810471 & -0.759609 \\
$\mathrm{H}$ & 2.038140 & 1.826638 & 0.334663 \\
$\mathrm{H}$ & -1.371264 & 2.304030 & 0.750559 \\
$\mathrm{H}$ & 0.050130 & 2.167919 & 1.799328 \\
$\mathrm{H}$ & 0.251414 & 2.833158 & -0.932092 \\
$\mathrm{H}$ & -2.899262 & 0.399987 & 1.162857 \\
$\mathrm{H}$ & -2.870557 & 0.999701 & -0.503005 \\
$\mathrm{H}$ & -2.695397 & -1.678733 & -0.484955
\end{tabular}

b) $\mathrm{B}_{6}^{-}(\mathrm{C} 7)$

Energies: $\mathrm{G}_{\mathrm{gas}}=-591.184781 \quad \mathrm{G}_{\mathrm{aq}}=-742.452546$

$\underline{\text { Coordinates }}$

$\begin{array}{crrr} & & & \\ \mathrm{N} & -1.273706 & 1.960874 & -0.077335 \\ \mathrm{C} & 0.040850 & 1.982479 & 0.088417 \\ \mathrm{C} & 0.890686 & 0.879345 & 0.275839 \\ \mathrm{C} & 0.235893 & -0.409554 & 0.295280 \\ \mathrm{C} & -1.126487 & -0.465281 & 0.107377 \\ \mathrm{C} & -1.939697 & 0.734933 & -0.084380 \\ \mathrm{C} & -3.303778 & 0.666401 & -0.258753 \\ \mathrm{O} & -1.808418 & -1.670837 & 0.106150\end{array}$




$\begin{array}{rrrr}\mathrm{C} & 1.001526 & -1.683660 & 0.553822 \\ \mathrm{O} & 0.739025 & -2.719963 & -0.440543 \\ \mathrm{C} & 2.355252 & 1.071223 & 0.437999 \\ \mathrm{O} & 3.157759 & 0.661318 & -0.727113 \\ \mathrm{H} & 0.496344 & 2.979746 & 0.080061 \\ \mathrm{H} & -3.821298 & -0.284372 & -0.265146 \\ \mathrm{H} & -3.870788 & 1.582803 & -0.386018 \\ \mathrm{H} & -1.162089 & -2.372983 & -0.080026 \\ \mathrm{H} & 2.078283 & -1.497645 & 0.595082 \\ \mathrm{H} & 0.695801 & -2.153470 & 1.497255 \\ \mathrm{H} & 0.895076 & -2.314846 & -1.305505 \\ \mathrm{H} & 2.559916 & 2.132582 & 0.641228 \\ \mathrm{H} & 2.797913 & 0.488778 & 1.257258 \\ \mathrm{H} & 2.614382 & 0.893836 & -1.494401\end{array}$

c) $\mathrm{B}_{6}{ }^{-}(\mathrm{O} 10)$

Energies: $\mathrm{G}_{\mathrm{gas}}=-591.236574 \quad \mathrm{G}_{\mathrm{aq}}=-591.510160$

$\underline{\text { Coordinates }}$

\begin{tabular}{lrrr} 
& $\mathrm{x}$ & $\mathrm{y}$ & \multicolumn{1}{c}{$\mathrm{z}$} \\
$\mathrm{C}$ & -0.247481 & 1.880498 & -0.047013 \\
$\mathrm{C}$ & -2.016492 & 0.403499 & 0.000000 \\
$\mathrm{C}$ & -1.164699 & -0.755256 & 0.259134 \\
$\mathrm{C}$ & 0.250145 & -0.466919 & 0.333577 \\
$\mathrm{C}$ & 0.686744 & 0.864410 & 0.192327 \\
$\mathrm{C}$ & -3.498631 & 0.161212 & -0.106187 \\
$\mathrm{C}$ & 1.198258 & -1.610250 & 0.599382 \\
$\mathrm{C}$ & 2.145029 & 1.235883 & 0.229086 \\
$\mathrm{O}$ & 2.096941 & -1.936642 & -0.484710 \\
$\mathrm{O}$ & -1.666502 & -1.914528 & 0.414470 \\
$\mathrm{O}$ & 2.859002 & 0.842033 & -0.980094 \\
$\mathrm{~N}$ & -1.573115 & 1.649720 & -0.143220 \\
$\mathrm{H}$ & 0.076600 & 2.916665 & -0.152392 \\
$\mathrm{H}$ & -3.720380 & -0.567528 & -0.897250 \\
$\mathrm{H}$ & -3.888658 & -0.283123 & 0.819499 \\
$\mathrm{H}$ & -4.015396 & 1.103534 & -0.312012 \\
$\mathrm{H}$ & 1.798491 & -1.414938 & 1.506685 \\
$\mathrm{H}$ & 0.596402 & -2.503818 & 0.772583 \\
$\mathrm{H}$ & 2.517323 & -1.111052 & -0.777645 \\
$\mathrm{H}$ & 2.256396 & 2.319920 & 0.367471 \\
$\mathrm{H}$ & 2.685385 & 0.731916 & 1.037047 \\
$\mathrm{H}$ & 2.272865 & 1.055019 & -1.720592
\end{tabular}




$$
\text { d) } B_{6}^{-}(011)
$$

Energies: $\mathrm{G}_{\mathrm{gas}}=-591.220124 \quad \mathrm{G}_{\mathrm{aq}}=-591.504029$

Coordinates

$\begin{array}{lrrr} & \mathrm{x} & \mathrm{y} & \mathrm{z} \\ \mathrm{C} & -0.245337 & 1.890919 & -0.072085 \\ \mathrm{C} & -2.005354 & 0.416447 & 0.000000 \\ \mathrm{C} & -1.110579 & -0.649810 & 0.210817 \\ \mathrm{C} & 0.272855 & -0.436650 & 0.292427 \\ \mathrm{C} & 0.713330 & 0.896900 & 0.133288 \\ \mathrm{C} & -3.490403 & 0.164347 & -0.082306 \\ \mathrm{C} & 1.288624 & -1.578469 & 0.500581 \\ \mathrm{C} & 2.192676 & 1.252214 & 0.162641 \\ \mathrm{O} & 2.212834 & -1.691387 & -0.488141 \\ \mathrm{O} & -1.676057 & -1.902750 & 0.337961 \\ \mathrm{O} & 2.926042 & 0.699578 & -0.904420 \\ \mathrm{~N} & -1.570358 & 1.675508 & -0.133143 \\ \mathrm{H} & 0.072171 & 2.926012 & -0.197800 \\ \mathrm{H} & -3.733188 & -0.525786 & -0.900112 \\ \mathrm{H} & -3.870531 & -0.298093 & 0.837554 \\ \mathrm{H} & -4.004689 & 1.114430 & -0.247525 \\ \mathrm{H} & -0.943593 & -2.536833 & 0.415960 \\ \mathrm{H} & 1.726253 & -1.406455 & 1.521751 \\ \mathrm{H} & 0.712644 & -2.538444 & 0.646628 \\ \mathrm{H} & 2.735388 & -0.323237 & -0.836729 \\ \mathrm{H} & 2.289041 & 2.346456 & 0.118825 \\ \mathrm{H} & 2.611594 & 0.934483 & 1.138082\end{array}$

e) $\mathrm{B}_{6}{ }^{-}(\mathrm{O} 12)$

Energies: $\mathrm{G}_{\mathrm{gas}}=-591.197706 \quad \mathrm{G}_{\mathrm{aq}}=-591.473665$

Coordinates

$\begin{array}{lrrr} & \mathrm{x} & \mathrm{y} & \mathrm{z} \\ \mathrm{C} & -1.869480 & -0.580129 & 0.023566 \\ \mathrm{~N} & -1.424244 & -1.834992 & -0.106795 \\ \mathrm{C} & -0.096467 & -2.030227 & -0.208229 \\ \mathrm{C} & 0.863606 & -1.015844 & -0.190009 \\ \mathrm{C} & 0.400595 & 0.307114 & -0.037403 \\ \mathrm{C} & -0.982909 & 0.515211 & 0.066075 \\ \mathrm{C} & 2.376616 & -1.299102 & -0.259883 \\ \mathrm{O} & 3.073533 & -0.708131 & 0.731731 \\ \mathrm{C} & 1.402038 & 1.431461 & 0.081871 \\ \mathrm{O} & 0.823662 & 2.670115 & -0.467015 \\ \mathrm{O} & -1.544879 & 1.762645 & 0.227925\end{array}$




$\begin{array}{lrrr}\mathrm{C} & -3.357431 & -0.358150 & 0.141901 \\ \mathrm{H} & 0.226494 & -3.066524 & -0.300262 \\ \mathrm{H} & -3.621381 & 0.110102 & 1.099188 \\ \mathrm{H} & -3.727667 & 0.312843 & -0.643697 \\ \mathrm{H} & -3.866468 & -1.322332 & 0.063831 \\ \mathrm{H} & -0.838924 & 2.414323 & 0.040303 \\ \mathrm{H} & 2.326789 & 1.186270 & -0.440209 \\ \mathrm{H} & 1.662959 & 1.586464 & 1.134856 \\ \mathrm{H} & 1.417136 & 3.393408 & -0.234213 \\ \mathrm{H} & 2.470947 & -2.419677 & -0.314598 \\ \mathrm{H} & 2.681874 & -0.968960 & -1.306104\end{array}$

f) $\cdot \mathrm{OH}_{2}{ }^{+}$

Energies: $\mathrm{G}_{\mathrm{gas}}=-75.968449 \quad \mathrm{G}_{\mathrm{aq}}=-76.148830$

Coordinates

$\begin{array}{cccc} & & & \\ & \mathrm{x} & \mathrm{y} & \mathrm{z} \\ \mathrm{O} & 0.000000 & 0.000000 & 0.114807 \\ \mathrm{H} & 0.000000 & 0.828575 & -0.459228 \\ \mathrm{H} & 0.000000 & -0.828575 & -0.459228\end{array}$

5.B $\mathrm{B}_{6}+\cdot$ OOH $----->\mathrm{B}_{6}^{-}+\cdot \mathrm{O}_{2} \mathrm{H}_{2}{ }^{+}$(5)

\subsection{REACTANTS}

a) Pyridoxine

Energies: $\mathrm{G}_{\mathrm{gas}}=-591.770767 \quad \mathrm{G}_{\mathrm{aq}}=-591.967495$

$\underline{\text { Coordinates }}$

\begin{tabular}{lrrr} 
& \multicolumn{1}{c}{$\mathrm{x}$} & $\mathrm{y}$ & \multicolumn{1}{c}{$\mathrm{z}$} \\
$\mathrm{C}$ & 0.010624 & 2.001714 & 0.073482 \\
$\mathrm{C}$ & -1.859630 & 0.662745 & -0.067344 \\
$\mathrm{C}$ & -1.060173 & -0.492804 & 0.093001 \\
$\mathrm{C}$ & 0.325908 & -0.373582 & 0.268084 \\
$\mathrm{C}$ & 0.880258 & 0.923474 & 0.247645 \\
$\mathrm{C}$ & -3.349532 & 0.537076 & -0.231889 \\
$\mathrm{C}$ & 1.159865 & -1.613381 & 0.525282 \\
$\mathrm{C}$ & 2.365388 & 1.175548 & 0.387882 \\
$\mathrm{O}$ & 0.780951 & -2.721212 & -0.321003 \\
$\mathrm{O}$ & -1.697245 & -1.697936 & 0.088458 \\
$\mathrm{O}$ & 3.133284 & 0.631117 & -0.697116 \\
$\mathrm{~N}$ & -1.316995 & 1.883493 & -0.072135
\end{tabular}




$\begin{array}{rrrr}\mathrm{H} & 0.402989 & 3.017553 & 0.053538 \\ \mathrm{H} & -3.599145 & -0.088961 & -1.096098 \\ \mathrm{H} & -3.804398 & 0.056403 & 0.642059 \\ \mathrm{H} & -3.781910 & 1.530398 & -0.363276 \\ \mathrm{H} & -1.030495 & -2.398743 & -0.054541 \\ \mathrm{H} & 2.224816 & -1.405823 & 0.409043 \\ \mathrm{H} & 0.986237 & -1.978769 & 1.544025 \\ \mathrm{H} & 1.091175 & -2.544588 & -1.219873 \\ \mathrm{H} & 2.543280 & 2.254822 & 0.482327 \\ \mathrm{H} & 2.775640 & 0.702345 & 1.285015 \\ \mathrm{H} & 2.838618 & 1.050425 & -1.516841\end{array}$

b) $\cdot \mathrm{OOH}$ Radical

Energies: $\mathrm{G}_{\mathrm{gas}}=-150.921387 \quad \mathrm{G}_{\mathrm{aq}}=-150.988356$

Coordinates

$\begin{array}{cccc} & \mathrm{x} & \mathrm{y} & \mathrm{z} \\ \mathrm{O} & 0.055545 & 0.721264 & 0.000000 \\ \mathrm{O} & 0.055545 & -0.612053 & 0.000000 \\ \mathrm{H} & -0.888716 & -0.873690 & 0.000000\end{array}$

\subsection{PRODUCTS}

a) $\mathrm{B}_{6}^{-}(\mathrm{C} 6)$

Energies: $\mathrm{G}_{\mathrm{gas}}=-591.158825 \quad \mathrm{G}_{\mathrm{aq}}=-591.429972$

$\underline{\text { Coordinates }}$

$\begin{array}{lrrr} & \mathrm{x} & \mathrm{y} & \mathrm{z} \\ \mathrm{C} & 1.195700 & 0.107914 & 0.149921 \\ \mathrm{C} & 1.381240 & -1.219399 & -0.250671 \\ \mathrm{~N} & 0.341434 & -2.039237 & -0.516066 \\ \mathrm{C} & -0.952785 & -1.606013 & -0.404691 \\ \mathrm{C} & -1.163834 & -0.269105 & 0.012859 \\ \mathrm{C} & -0.109289 & 0.609312 & 0.299976 \\ \mathrm{C} & 2.776841 & -1.781249 & -0.398778 \\ \mathrm{C} & -2.647417 & 0.112903 & 0.123223 \\ \mathrm{O} & -3.434817 & -0.978625 & -0.283853 \\ \mathrm{C} & -0.312457 & 2.026325 & 0.773718 \\ \mathrm{O} & 0.456691 & 2.996404 & 0.000000 \\ \mathrm{O} & 2.315627 & 0.897259 & 0.403931 \\ \mathrm{H} & 3.379838 & -1.193883 & -1.104079\end{array}$




$\begin{array}{lrrr}\mathrm{H} & 3.322450 & -1.778119 & 0.554863 \\ \mathrm{H} & 2.696471 & -2.810471 & -0.759609 \\ \mathrm{H} & 2.038140 & 1.826638 & 0.334663 \\ \mathrm{H} & -1.371264 & 2.304030 & 0.750559 \\ \mathrm{H} & 0.050130 & 2.167919 & 1.799328 \\ \mathrm{H} & 0.251414 & 2.833158 & -0.932092 \\ \mathrm{H} & -2.899262 & 0.399987 & 1.162857 \\ \mathrm{H} & -2.870557 & 0.999701 & -0.503005 \\ \mathrm{H} & -2.695397 & -1.678733 & -0.484955\end{array}$

b) $\mathrm{B}_{6}^{-}(\mathrm{C} 7)$

Energies: $\mathrm{G}_{\mathrm{gas}}=-591.184781 \quad \mathrm{G}_{\mathrm{aq}}=-742.452546$

$\underline{\text { Coordinates }}$

\begin{tabular}{lrrr} 
& \multicolumn{1}{c}{$\mathrm{x}$} & $\mathrm{y}$ & \multicolumn{1}{c}{$\mathrm{z}$} \\
$\mathrm{N}$ & -1.273706 & 1.960874 & -0.077335 \\
$\mathrm{C}$ & 0.040850 & 1.982479 & 0.088417 \\
$\mathrm{C}$ & 0.890686 & 0.879345 & 0.275839 \\
$\mathrm{C}$ & 0.235893 & -0.409554 & 0.295280 \\
$\mathrm{C}$ & -1.126487 & -0.465281 & 0.107377 \\
$\mathrm{C}$ & -1.939697 & 0.734933 & -0.084380 \\
$\mathrm{C}$ & -3.303778 & 0.666401 & -0.258753 \\
$\mathrm{O}$ & -1.808418 & -1.670837 & 0.106150 \\
$\mathrm{C}$ & 1.001526 & -1.683660 & 0.553822 \\
$\mathrm{O}$ & 0.739025 & -2.719963 & -0.440543 \\
$\mathrm{C}$ & 2.355252 & 1.071223 & 0.437999 \\
$\mathrm{O}$ & 3.157759 & 0.661318 & -0.727113 \\
$\mathrm{H}$ & 0.496344 & 2.979746 & 0.080061 \\
$\mathrm{H}$ & -3.821298 & -0.284372 & -0.265146 \\
$\mathrm{H}$ & -3.870788 & 1.582803 & -0.386018 \\
$\mathrm{H}$ & -1.162089 & -2.372983 & -0.080026 \\
$\mathrm{H}$ & 2.078283 & -1.497645 & 0.595082 \\
$\mathrm{H}$ & 0.695801 & -2.153470 & 1.497255 \\
$\mathrm{H}$ & 0.895076 & -2.314846 & -1.305505 \\
$\mathrm{H}$ & 2.559916 & 2.132582 & 0.641228 \\
$\mathrm{H}$ & 2.797913 & 0.488778 & 1.257258 \\
$\mathrm{H}$ & 2.614382 & 0.893836 & -1.494401
\end{tabular}

c) $\mathrm{B}_{6}{ }^{-}(\mathrm{O} 10)$

Energies: $\mathrm{G}_{\mathrm{gas}}=-591.236574 \quad \mathrm{G}_{\mathrm{aq}}=-591.510160$

$\underline{\text { Coordinates }}$

$\begin{array}{lll}\mathrm{x} & \mathrm{y}\end{array}$




$\begin{array}{lrrr}\mathrm{C} & -0.247481 & 1.880498 & -0.047013 \\ \mathrm{C} & -2.016492 & 0.403499 & 0.000000 \\ \mathrm{C} & -1.164699 & -0.755256 & 0.259134 \\ \mathrm{C} & 0.250145 & -0.466919 & 0.333577 \\ \mathrm{C} & 0.686744 & 0.864410 & 0.192327 \\ \mathrm{C} & -3.498631 & 0.161212 & -0.106187 \\ \mathrm{C} & 1.198258 & -1.610250 & 0.599382 \\ \mathrm{C} & 2.145029 & 1.235883 & 0.229086 \\ \mathrm{O} & 2.096941 & -1.936642 & -0.484710 \\ \mathrm{O} & -1.666502 & -1.914528 & 0.414470 \\ \mathrm{O} & 2.859002 & 0.842033 & -0.980094 \\ \mathrm{~N} & -1.573115 & 1.649720 & -0.143220 \\ \mathrm{H} & 0.076600 & 2.916665 & -0.152392 \\ \mathrm{H} & -3.720380 & -0.567528 & -0.897250 \\ \mathrm{H} & -3.888658 & -0.283123 & 0.819499 \\ \mathrm{H} & -4.015396 & 1.103534 & -0.312012 \\ \mathrm{H} & 1.798491 & -1.414938 & 1.506685 \\ \mathrm{H} & 0.596402 & -2.503818 & 0.772583 \\ \mathrm{H} & 2.517323 & -1.111052 & -0.777645 \\ \mathrm{H} & 2.256396 & 2.319920 & 0.367471 \\ \mathrm{H} & 2.685385 & 0.731916 & 1.037047 \\ \mathrm{H} & 2.272865 & 1.055019 & -1.720592\end{array}$

d) $\mathrm{B}_{6}^{-}(\mathrm{O} 11)$

Energies: $\mathrm{G}_{\mathrm{gas}}=-591.220124 \quad \mathrm{G}_{\mathrm{aq}}=-591.504029$

$\underline{\text { Coordinates }}$

$\begin{array}{lrrr} & \mathrm{x} & \mathrm{y} & \mathrm{z} \\ \mathrm{C} & -0.245337 & 1.890919 & -0.072085 \\ \mathrm{C} & -2.005354 & 0.416447 & 0.000000 \\ \mathrm{C} & -1.110579 & -0.649810 & 0.210817 \\ \mathrm{C} & 0.272855 & -0.436650 & 0.292427 \\ \mathrm{C} & 0.713330 & 0.896900 & 0.133288 \\ \mathrm{C} & -3.490403 & 0.164347 & -0.082306 \\ \mathrm{C} & 1.288624 & -1.578469 & 0.500581 \\ \mathrm{C} & 2.192676 & 1.252214 & 0.162641 \\ \mathrm{O} & 2.212834 & -1.691387 & -0.488141 \\ \mathrm{O} & -1.676057 & -1.902750 & 0.337961 \\ \mathrm{O} & 2.926042 & 0.699578 & -0.904420 \\ \mathrm{~N} & -1.570358 & 1.675508 & -0.133143 \\ \mathrm{H} & 0.072171 & 2.926012 & -0.197800 \\ \mathrm{H} & -3.733188 & -0.525786 & -0.900112 \\ \mathrm{H} & -3.870531 & -0.298093 & 0.837554 \\ \mathrm{H} & -4.004689 & 1.114430 & -0.247525 \\ \mathrm{H} & -0.943593 & -2.536833 & 0.415960\end{array}$




$\begin{array}{lrrr}\mathrm{H} & 1.726253 & -1.406455 & 1.521751 \\ \mathrm{H} & 0.712644 & -2.538444 & 0.646628 \\ \mathrm{H} & 2.735388 & -0.323237 & -0.836729 \\ \mathrm{H} & 2.289041 & 2.346456 & 0.118825 \\ \mathrm{H} & 2.611594 & 0.934483 & 1.138082\end{array}$

e) $\mathrm{B}_{6}^{-}(\mathrm{O} 12)$

Energies: $\mathrm{G}_{\mathrm{gas}}=-591.197706 \quad \mathrm{G}_{\mathrm{aq}}=-591.473665$

Coordinates

\begin{tabular}{lrrr} 
& \multicolumn{1}{c}{$\mathrm{x}$} & $\mathrm{y}$ & $\mathrm{z}$ \\
$\mathrm{C}$ & -1.869480 & -0.580129 & 0.023566 \\
$\mathrm{~N}$ & -1.424244 & -1.834992 & -0.106795 \\
$\mathrm{C}$ & -0.096467 & -2.030227 & -0.208229 \\
$\mathrm{C}$ & 0.863606 & -1.015844 & -0.190009 \\
$\mathrm{C}$ & 0.400595 & 0.307114 & -0.037403 \\
$\mathrm{C}$ & -0.982909 & 0.515211 & 0.066075 \\
$\mathrm{C}$ & 2.376616 & -1.299102 & -0.259883 \\
$\mathrm{O}$ & 3.073533 & -0.708131 & 0.731731 \\
$\mathrm{C}$ & 1.402038 & 1.431461 & 0.081871 \\
$\mathrm{O}$ & 0.823662 & 2.670115 & -0.467015 \\
$\mathrm{O}$ & -1.544879 & 1.762645 & 0.227925 \\
$\mathrm{C}$ & -3.357431 & -0.358150 & 0.141901 \\
$\mathrm{H}$ & 0.226494 & -3.066524 & -0.300262 \\
$\mathrm{H}$ & -3.621381 & 0.110102 & 1.099188 \\
$\mathrm{H}$ & -3.727667 & 0.312843 & -0.643697 \\
$\mathrm{H}$ & -3.866468 & -1.322332 & 0.063831 \\
$\mathrm{H}$ & -0.838924 & 2.414323 & 0.040303 \\
$\mathrm{H}$ & 2.326789 & 1.186270 & -0.440209 \\
$\mathrm{H}$ & 1.662959 & 1.586464 & 1.134856 \\
$\mathrm{H}$ & 1.417136 & 3.393408 & -0.234213 \\
$\mathrm{H}$ & 2.470947 & -2.419677 & -0.314598 \\
$\mathrm{H}$ & 2.681874 & -0.968960 & -1.306104
\end{tabular}

$$
\text { f) } \cdot \mathrm{O}_{2} \mathrm{H}_{2}{ }^{+}
$$

Energies: $\mathrm{G}_{\mathrm{gas}}=-151.163807 \quad \mathrm{G}_{\mathrm{aq}}=-151.366972$

$\underline{\text { Coordinates }}$

$\begin{array}{crrc} & \mathrm{x} & \mathrm{y} & \mathrm{z} \\ \mathrm{O} & 0.000000 & 0.657691 & 0.000000 \\ \mathrm{O} & 0.000000 & -0.657691 & 0.000000 \\ \mathrm{H} & 0.968376 & 0.907817 & 0.000000 \\ \mathrm{H} & -0.968376 & -0.907817 & 0.000000\end{array}$




\section{6. $\mathrm{B}_{6}+\cdot \mathrm{O}_{2}^{-}----->\mathrm{B}_{6}^{-}+\cdot 0 \mathrm{OH}$ (6)}

\subsection{REACTANTS}

a) Pyridoxine

Energies: $\mathrm{G}_{\mathrm{gas}}=-591.770767 \quad \mathrm{G}_{\mathrm{aq}}=-591.967495$

$\underline{\text { Coordinates }}$

\begin{tabular}{lrrr} 
& \multicolumn{1}{c}{$\mathrm{x}$} & $\mathrm{y}$ & \multicolumn{1}{c}{$\mathrm{z}$} \\
$\mathrm{C}$ & 0.010624 & 2.001714 & 0.073482 \\
$\mathrm{C}$ & -1.859630 & 0.662745 & -0.067344 \\
$\mathrm{C}$ & -1.060173 & -0.492804 & 0.093001 \\
$\mathrm{C}$ & 0.325908 & -0.373582 & 0.268084 \\
$\mathrm{C}$ & 0.880258 & 0.923474 & 0.247645 \\
$\mathrm{C}$ & -3.349532 & 0.537076 & -0.231889 \\
$\mathrm{C}$ & 1.159865 & -1.613381 & 0.525282 \\
$\mathrm{C}$ & 2.365388 & 1.175548 & 0.387882 \\
$\mathrm{O}$ & 0.780951 & -2.721212 & -0.321003 \\
$\mathrm{O}$ & -1.697245 & -1.697936 & 0.088458 \\
$\mathrm{O}$ & 3.133284 & 0.631117 & -0.697116 \\
$\mathrm{~N}$ & -1.316995 & 1.883493 & -0.072135 \\
$\mathrm{H}$ & 0.402989 & 3.017553 & 0.053538 \\
$\mathrm{H}$ & -3.599145 & -0.088961 & -1.096098 \\
$\mathrm{H}$ & -3.804398 & 0.056403 & 0.642059 \\
$\mathrm{H}$ & -3.781910 & 1.530398 & -0.363276 \\
$\mathrm{H}$ & -1.030495 & -2.398743 & -0.054541 \\
$\mathrm{H}$ & 2.224816 & -1.405823 & 0.409043 \\
$\mathrm{H}$ & 0.986237 & -1.978769 & 1.544025 \\
$\mathrm{H}$ & 1.091175 & -2.544588 & -1.219873 \\
$\mathrm{H}$ & 2.543280 & 2.254822 & 0.482327 \\
$\mathrm{H}$ & 2.775640 & 0.702345 & 1.285015 \\
$\mathrm{H}$ & 2.838618 & 1.050425 & -1.516841
\end{tabular}

b) $\cdot \mathrm{O}_{2}^{-}$Radical

Energies: $\mathrm{G}_{\mathrm{gas}}=-150.364299 \quad \mathrm{G}_{\mathrm{aq}}=-150.535287$

$\underline{\text { Coordinates }}$
$\begin{array}{cccc} & \mathrm{x} & \mathrm{y} & \mathrm{z} \\ \mathrm{O} & 0.000000 & 0.000000 & 0.675349\end{array}$
$\begin{array}{llll}\mathrm{O} & 0.000000 & 0.000000 & -0.675349\end{array}$ 


\subsection{PRODUCTS}

a) $\mathrm{B}_{6}^{-}(\mathrm{C} 6)$

Energies: $\mathrm{G}_{\mathrm{gas}}=-591.158825 \quad \mathrm{G}_{\mathrm{aq}}=-591.429972$

$\underline{\text { Coordinates }}$

\begin{tabular}{lrrr} 
& \multicolumn{1}{c}{$\mathrm{x}$} & $\mathrm{y}$ & $\mathrm{z}$ \\
$\mathrm{C}$ & 1.195700 & 0.107914 & 0.149921 \\
$\mathrm{C}$ & 1.381240 & -1.219399 & -0.250671 \\
$\mathrm{~N}$ & 0.341434 & -2.039237 & -0.516066 \\
$\mathrm{C}$ & -0.952785 & -1.606013 & -0.404691 \\
$\mathrm{C}$ & -1.163834 & -0.269105 & 0.012859 \\
$\mathrm{C}$ & -0.109289 & 0.609312 & 0.299976 \\
$\mathrm{C}$ & 2.776841 & -1.781249 & -0.398778 \\
$\mathrm{C}$ & -2.647417 & 0.112903 & 0.123223 \\
$\mathrm{O}$ & -3.434817 & -0.978625 & -0.283853 \\
$\mathrm{C}$ & -0.312457 & 2.026325 & 0.773718 \\
$\mathrm{O}$ & 0.456691 & 2.996404 & 0.000000 \\
$\mathrm{O}$ & 2.315627 & 0.897259 & 0.403931 \\
$\mathrm{H}$ & 3.379838 & -1.193883 & -1.104079 \\
$\mathrm{H}$ & 3.322450 & -1.778119 & 0.554863 \\
$\mathrm{H}$ & 2.696471 & -2.810471 & -0.759609 \\
$\mathrm{H}$ & 2.038140 & 1.826638 & 0.334663 \\
$\mathrm{H}$ & -1.371264 & 2.304030 & 0.750559 \\
$\mathrm{H}$ & 0.050130 & 2.167919 & 1.799328 \\
$\mathrm{H}$ & 0.251414 & 2.833158 & -0.932092 \\
$\mathrm{H}$ & -2.899262 & 0.399987 & 1.162857 \\
$\mathrm{H}$ & -2.870557 & 0.999701 & -0.503005 \\
$\mathrm{H}$ & -2.695397 & -1.678733 & -0.484955
\end{tabular}

b) $\mathrm{B}_{6}^{-}(\mathrm{C} 7)$

Energies: $\mathrm{G}_{\mathrm{gas}}=-591.184781 \quad \mathrm{G}_{\mathrm{aq}}=-742.452546$

$\underline{\text { Coordinates }}$

$\begin{array}{cccc} & \mathrm{x} & \mathrm{y} & \mathrm{z} \\ \mathrm{N} & -1.273706 & 1.960874 & -0.077335 \\ \mathrm{C} & 0.040850 & 1.982479 & 0.088417 \\ \mathrm{C} & 0.890686 & 0.879345 & 0.275839 \\ \mathrm{C} & 0.235893 & -0.409554 & 0.295280 \\ \mathrm{C} & -1.126487 & -0.465281 & 0.107377 \\ \mathrm{C} & -1.939697 & 0.734933 & -0.084380 \\ \mathrm{C} & -3.303778 & 0.666401 & -0.258753 \\ \mathrm{O} & -1.808418 & -1.670837 & 0.106150\end{array}$




$\begin{array}{rrrr}\mathrm{C} & 1.001526 & -1.683660 & 0.553822 \\ \mathrm{O} & 0.739025 & -2.719963 & -0.440543 \\ \mathrm{C} & 2.355252 & 1.071223 & 0.437999 \\ \mathrm{O} & 3.157759 & 0.661318 & -0.727113 \\ \mathrm{H} & 0.496344 & 2.979746 & 0.080061 \\ \mathrm{H} & -3.821298 & -0.284372 & -0.265146 \\ \mathrm{H} & -3.870788 & 1.582803 & -0.386018 \\ \mathrm{H} & -1.162089 & -2.372983 & -0.080026 \\ \mathrm{H} & 2.078283 & -1.497645 & 0.595082 \\ \mathrm{H} & 0.695801 & -2.153470 & 1.497255 \\ \mathrm{H} & 0.895076 & -2.314846 & -1.305505 \\ \mathrm{H} & 2.559916 & 2.132582 & 0.641228 \\ \mathrm{H} & 2.797913 & 0.488778 & 1.257258 \\ \mathrm{H} & 2.614382 & 0.893836 & -1.494401\end{array}$

c) $\mathrm{B}_{6}{ }^{-}(\mathrm{O} 10)$

Energies: $\mathrm{G}_{\mathrm{gas}}=-591.236574 \quad \mathrm{G}_{\mathrm{aq}}=-591.510160$

$\underline{\text { Coordinates }}$

\begin{tabular}{lrrr} 
& \multicolumn{1}{c}{$\mathrm{x}$} & $\mathrm{y}$ & \multicolumn{1}{c}{$\mathrm{z}$} \\
$\mathrm{C}$ & -0.247481 & 1.880498 & -0.047013 \\
$\mathrm{C}$ & -2.016492 & 0.403499 & 0.000000 \\
$\mathrm{C}$ & -1.164699 & -0.755256 & 0.259134 \\
$\mathrm{C}$ & 0.250145 & -0.466919 & 0.333577 \\
$\mathrm{C}$ & 0.686744 & 0.864410 & 0.192327 \\
$\mathrm{C}$ & -3.498631 & 0.161212 & -0.106187 \\
$\mathrm{C}$ & 1.198258 & -1.610250 & 0.599382 \\
$\mathrm{C}$ & 2.145029 & 1.235883 & 0.229086 \\
$\mathrm{O}$ & 2.096941 & -1.936642 & -0.484710 \\
$\mathrm{O}$ & -1.666502 & -1.914528 & 0.414470 \\
$\mathrm{O}$ & 2.859002 & 0.842033 & -0.980094 \\
$\mathrm{~N}$ & -1.573115 & 1.649720 & -0.143220 \\
$\mathrm{H}$ & 0.076600 & 2.916665 & -0.152392 \\
$\mathrm{H}$ & -3.720380 & -0.567528 & -0.897250 \\
$\mathrm{H}$ & -3.888658 & -0.283123 & 0.819499 \\
$\mathrm{H}$ & -4.015396 & 1.103534 & -0.312012 \\
$\mathrm{H}$ & 1.798491 & -1.414938 & 1.506685 \\
$\mathrm{H}$ & 0.596402 & -2.503818 & 0.772583 \\
$\mathrm{H}$ & 2.517323 & -1.111052 & -0.777645 \\
$\mathrm{H}$ & 2.256396 & 2.319920 & 0.367471 \\
$\mathrm{H}$ & 2.685385 & 0.731916 & 1.037047 \\
$\mathrm{H}$ & 2.272865 & 1.055019 & -1.720592
\end{tabular}

d) $\mathrm{B}_{6}{ }^{-}(\mathrm{O} 11)$ 
Energies: $\mathrm{G}_{\mathrm{gas}}=-591.220124 \quad \mathrm{G}_{\mathrm{aq}}=-591.504029$

$\underline{\text { Coordinates }}$

\begin{tabular}{lrrr} 
& \multicolumn{1}{c}{$\mathrm{x}$} & $\mathrm{y}$ & \multicolumn{1}{c}{$\mathrm{z}$} \\
$\mathrm{C}$ & -0.245337 & 1.890919 & -0.072085 \\
$\mathrm{C}$ & -2.005354 & 0.416447 & 0.000000 \\
$\mathrm{C}$ & -1.110579 & -0.649810 & 0.210817 \\
$\mathrm{C}$ & 0.272855 & -0.436650 & 0.292427 \\
$\mathrm{C}$ & 0.713330 & 0.896900 & 0.133288 \\
$\mathrm{C}$ & -3.490403 & 0.164347 & -0.082306 \\
$\mathrm{C}$ & 1.288624 & -1.578469 & 0.500581 \\
$\mathrm{C}$ & 2.192676 & 1.252214 & 0.162641 \\
$\mathrm{O}$ & 2.212834 & -1.691387 & -0.488141 \\
$\mathrm{O}$ & -1.676057 & -1.902750 & 0.337961 \\
$\mathrm{O}$ & 2.926042 & 0.699578 & -0.904420 \\
$\mathrm{~N}$ & -1.570358 & 1.675508 & -0.133143 \\
$\mathrm{H}$ & 0.072171 & 2.926012 & -0.197800 \\
$\mathrm{H}$ & -3.733188 & -0.525786 & -0.900112 \\
$\mathrm{H}$ & -3.870531 & -0.298093 & 0.837554 \\
$\mathrm{H}$ & -4.004689 & 1.114430 & -0.247525 \\
$\mathrm{H}$ & -0.943593 & -2.536833 & 0.415960 \\
$\mathrm{H}$ & 1.726253 & -1.406455 & 1.521751 \\
$\mathrm{H}$ & 0.712644 & -2.538444 & 0.646628 \\
$\mathrm{H}$ & 2.735388 & -0.323237 & -0.836729 \\
$\mathrm{H}$ & 2.289041 & 2.346456 & 0.118825 \\
$\mathrm{H}$ & 2.611594 & 0.934483 & 1.138082
\end{tabular}

e) $\mathrm{B}_{6}^{-}(\mathrm{O} 12)$

Energies: $\mathrm{G}_{\mathrm{gas}}=-591.197706 \quad \mathrm{G}_{\mathrm{aq}}=-591.473665$

$\underline{\text { Coordinates }}$

$\begin{array}{lrrr} & \mathrm{x} & \mathrm{y} & \mathrm{z} \\ \mathrm{C} & -1.869480 & -0.580129 & 0.023566 \\ \mathrm{~N} & -1.424244 & -1.834992 & -0.106795 \\ \mathrm{C} & -0.096467 & -2.030227 & -0.208229 \\ \mathrm{C} & 0.863606 & -1.015844 & -0.190009 \\ \mathrm{C} & 0.400595 & 0.307114 & -0.037403 \\ \mathrm{C} & -0.982909 & 0.515211 & 0.066075 \\ \mathrm{C} & 2.376616 & -1.299102 & -0.259883 \\ \mathrm{O} & 3.073533 & -0.708131 & 0.731731 \\ \mathrm{C} & 1.402038 & 1.431461 & 0.081871 \\ \mathrm{O} & 0.823662 & 2.670115 & -0.467015 \\ \mathrm{O} & -1.544879 & 1.762645 & 0.227925 \\ \mathrm{C} & -3.357431 & -0.358150 & 0.141901\end{array}$


$\mathrm{H} \quad 0.226494 \quad-3.066524 \quad-0.300262$

$\mathrm{H} \quad-3.621381 \quad 0.110102 \quad 1.099188$

$\mathrm{H} \quad-3.727667 \quad 0.312843 \quad-0.643697$

$\mathrm{H} \quad-3.866468 \quad-1.322332 \quad 0.063831$

$\mathrm{H} \quad-0.838924 \quad 2.414323 \quad 0.040303$

$\mathrm{H} \quad 2.326789 \quad 1.186270 \quad-0.440209$

$\mathrm{H} \quad 1.662959 \quad 1.586464 \quad 1.134856$

$\mathrm{H} \quad \begin{array}{llll}1.417136 & 3.393408 & -0.234213\end{array}$

$\mathrm{H} \quad 2.470947 \quad-2.419677 \quad-0.314598$

$\mathrm{H} \quad 2.681874 \quad-0.968960 \quad-1.306104$

f) $\cdot \mathrm{OOH}$ Radical

Energies: $\mathrm{G}_{\mathrm{gas}}=-150.921387 \quad \mathrm{G}_{\mathrm{aq}}=-150.988356$

$\underline{\text { Coordinates }}$

$\begin{array}{cccc} & \mathrm{x} & \mathrm{y} & \mathrm{z} \\ \mathrm{O} & 0.055545 & 0.721264 & 0.000000 \\ \mathrm{O} & 0.055545 & -0.612053 & 0.000000 \\ \mathrm{H} & -0.888716 & -0.873690 & 0.000000\end{array}$ 